\title{
Genomic transcriptional profiling identifies a candidate blood biomarker signature for the diagnosis of septicemic melioidosis Rungnapa Pankla* ${ }^{*}$, Surachat Buddhisa* ${ }^{*}$ Matthew Berry ${ }^{\ddagger}$, Derek M Blankenship§, Gregory J Bancroft", Jacques Banchereau ${ }^{\dagger}$, Ganjana Lertmemongkolchai ${ }^{*}$ and Damien Chaussabel ${ }^{\dagger}$
}

\begin{abstract}
Addresses: *Department of Clinical Immunology, Centre for Research and Development of Medical Diagnostic Laboratories, Faculty of Associated Medical Sciences, Khon Kaen University, 123 Mittraparp Road, Khon Kaen, 40002, Thailand. ${ }^{\dagger}$ Baylor-National Institute of Allergy and Infectious Diseases (NIAID), Cooperative Center for Translational Research on Human Immunology and Biodefense, Baylor Institute for Immunology Research and Baylor Research Institute, 3434 Live Oak St, Dallas, Texas, 75204, USA. *Division of Immunoregulation, National Institute for Medical Research, The Ridgeway, Mill Hill, London, NW7 1AA, UK. §Institute for Health Care Research and Improvement, Baylor Health Care System, 8080 N. Central Expressway Suite 500, Dallas, Texas, 75206, USA. "Department of Infectious and Tropical Diseases, London School of Hygiene and Tropical Medicine, Keppel St, London, WC1E 7HT, UK.
\end{abstract}

Correspondence: Ganjana Lertmemongkolchai. Email: ganja_le@kku.ac.th. Damien Chaussabel. Email: DamienC@BaylorHealth.edu

Published: 10 November 2009

Genome Biology 2009, 10:RI27 (doi:10.1 I86/gb-2009-10-II-r 127)

The electronic version of this article is the complete one and can be found online at http://genomebiology.com/2009//0/I I/R I 27
Received: 19 April 2009

Revised: 7 September 2009

Accepted: 10 November 2009

(c) 2009 Pankla et al.; licensee BioMed Central Ltd.

This is an open access article distributed under the terms of the Creative Commons Attribution License (http://creativecommons.org/licenses/by/2.0), which permits unrestricted use, distribution, and reproduction in any medium, provided the original work is properly cited.

\begin{abstract}
Background: Melioidosis is a severe infectious disease caused by Burkholderia pseudomallei, a Gram-negative bacillus classified by the National Institute of Allergy and Infectious Diseases (NIAID) as a category B priority agent. Septicemia is the most common presentation of the disease with a $40 \%$ mortality rate even with appropriate treatments. Better diagnostic tests are therefore needed to improve therapeutic efficacy and survival rates.

Results: We have used microarray technology to generate genome-wide transcriptional profiles (>48,000 transcripts) from the whole blood of patients with septicemic melioidosis $(n=32)$, patients with sepsis caused by other pathogens $(n=31)$, and uninfected controls $(n=29)$. Unsupervised analyses demonstrated the existence of a whole blood transcriptional signature distinguishing patients with sepsis from control subjects. The majority of changes observed were common to both septicemic melioidosis and sepsis caused by other infections, including genes related to inflammation, interferon-related genes, neutrophils, cytotoxic cells, and T-cells. Finally, class prediction analysis identified a 37 transcript candidate diagnostic signature that distinguished melioidosis from sepsis caused by other organisms with $100 \%$ accuracy in a training set. This finding was confirmed in 2 independent validation sets, which gave high prediction accuracies of $78 \%$ and $80 \%$, respectively. This signature was significantly enriched in genes coding for products involved in the MHC class II antigen processing and presentation pathway.

Conclusions: Blood transcriptional patterns distinguish patients with septicemic melioidosis from patients with sepsis caused by other pathogens. Once confirmed in a large scale trial this diagnostic signature might constitute the basis of a differential diagnostic assay.
\end{abstract}




\section{Background}

Melioidosis is an infectious disease caused by the Gram-negative bacillus Burkholderia pseudomallei. The disease is endemic in northern Australia, Southeast Asia, and northeast Thailand, where it is a common cause of community-acquired sepsis $[1,2]$. Cases of melioidosis have also been reported from other regions around the world [3]. In Thailand, the incidence rate of melioidosis was estimated as 4.4 cases per 100,000 individuals, but melioidosis cases are underreported due to a lack of adequate laboratory testing $[1,4]$. The disease is the leading cause of community-acquired septicemia in northeast Thailand [5]. The common clinical manifestation of melioidosis at initial presentation is febrile illness with pneumonia, which makes it difficult to distinguish from other infections [1,6]. However, in contrast to other infections, the majority of melioidosis patients develop sepsis rapidly after presentation, and the disease has a mortality rate of $40 \%$ despite appropriate treatment [6].

Definitive diagnosis requires isolation of $B$. pseudomallei from clinical specimens [1,7-9]. However, the rate of positive cultures is low and it may take up to a week to confirm a microbiological diagnosis of melioidosis, which can delay the initiation of appropriate therapy [1,10-12]. Antibody detection by indirect hemagglutination assay is faster than culture but lacks sensitivity and specificity, especially when used in an endemic area since most of the population is seropositive [1]. Amplification approaches to detect pathogen-specific genes by PCR have similarly shown variable specificity and sensitivity [7-9]. Missed or delayed diagnosis may have dire consequences since several antibiotics commonly used for Gram-negative septicemia are ineffective against $B$. pseudomallei $[1,3,13]$. It has been reported that faster diagnosis of other bloodstream infections permits earlier implementation of appropriate antimicrobial therapy and reduces mortality [14]. Animal models support the notion that an earlier diagnosis of melioidosis leads to an improved disease outcome, with increased survival observed when $B$. pseudomalleiinfected mice are treated with the appropriate antibiotics within 24 hours post-infection [15]. Thus, there is an urgent need for improved, rapid diagnostic tests for septicemic melioidosis and indicators of clinical severity $[1,6,10]$. Furthermore, B. pseudomallei has been classified as a category B agent of bioterrorism by the US Centers for Disease Control and Prevention and the National Institute of Allergy and Infectious Diseases (NIAID) due to its ability to initiate infection via aerosol contact; the rapid onset of sepsis following the development of symptoms and the high mortality rate even with medical treatment [16]. Taken together, these facts delineate the importance of developing novel tools for the rapid and definitive diagnosis of $B$. pseudomallei infection.

Microarray-based profiling of tumoral tissue has proved instrumental for the discovery of transcriptional biomarker signatures in patients with cancer [17]. The immune status of a patient can be assessed through the profiling of peripheral blood, which constitutes an accessible source of immune cells that migrate to and from sites of infection, and are exposed to pathogen as well as host-derived factors released in the circulation. Furthermore, through the analysis of whole blood it is possible to measure transcriptional responses caused by disease with minimal sampling bias or ex vivo manipulation. The use of gene expression microarrays as a tool to study the expression profiles of human blood has been reported in systemic autoimmune diseases and infectious diseases, including malaria, acute dengue hemorrhagic fever, febrile respiratory illness, and influenza A virus or bacterial infections [18-22]. In addition, previous studies have shown that microarray-based approaches allow researchers to identify blood expression profiles restricted to sepsis [23-25]. In the context of the present study, we have used a microarraybased approach to generate blood transcriptional profiles of septic patients who were recruited in northeast Thailand. After establishing a blood signature of sepsis, we developed a candidate biomarker signature that distinguishes B. pseudomallei from other infectious agents causing septicemia.

\section{Results \\ Patient characteristics}

A total of 598 subjects consisting of 29 uninfected controls and 569 patients diagnosed with sepsis were enrolled in this study and all subjects were Asian (Figure 1a). Of these 569 patients, 63 had positive blood cultures (32 grew B. pseudomallei and 31 grew other organisms) and were thus selected for microarray analysis. Meanwhile, 29 uninfected controls recruited in this study were 8 healthy donors, 12 patients with type 2 diabetes (T2D) and 9 patients who had recovered from melioidosis. Whole blood samples collected from these 29 uninfected controls and 63 septic patients were extracted for RNA in 3 separated experiments: the first set (34 samples) was assigned to a training set used for discovery; the second set (33 samples) was assigned to a first test set to independently evaluate the performance of candidate markers; and the third set ( 25 samples) was assigned to a second independent test set for further validation (Figure $1 \mathrm{~b}$ and Table 1).

The training set is composed of 34 samples: 24 patients with sepsis, all with positive blood cultures, including 11 patients with septicemic melioidosis; 13 patients with sepsis due to other organisms (1 Acinetobacter baumannii, 2 Corynebacterium spp., 3 Candida albicans, 3 Escherichia coli, 1 Salmonella serotype B, 1 Salmonella spp., 1 Staphylococcus aureus, and 1 non-group A or B Streptococcus); and 10 subjects from the same endemic area recruited as non-infected controls. These non-infected controls comprised 5 patients with T2D, a risk factor for melioidosis, and 5 patients with melioidosis who have recovered after complete treatment, and been followed up for at least 20 weeks without any sign of infection; 3 out of these 5 subjects were diabetic. Demographic, clinical 
Table I

\begin{tabular}{|c|c|c|c|c|c|}
\hline & Septicemic melioidosis & Other sepsis & Recovery & Type 2 diabetes & Healthy \\
\hline \multicolumn{6}{|l|}{ Training set $(n=34)$} \\
\hline Number of subjects & 11 & 13 & 5 & 5 & \\
\hline Mean age in years (range) & $54(4 \mid-70)$ & $56(37-74)$ & $46(4 \mid-64)$ & $40(39-68)$ & \\
\hline Sex (male/female) & $7 / 4$ & $4 / 9$ & $3 / 2$ & $\mathrm{I} / 4$ & \\
\hline Survivors/non-survivors & $6 / 5$ & $11 / 2$ & & & \\
\hline \multirow[t]{8}{*}{ Organisms (n) } & B. pseudomallei (I I) & A. baumannii (I) & & & \\
\hline & & Corynebacterium spp. (2) & & & \\
\hline & & C. albicans (3) & & & \\
\hline & & E. coli $(3)$ & & & \\
\hline & & Salmonella serotype B (I) & & & \\
\hline & & S. aureus (I) & & & \\
\hline & & Salmonella spp. (I) & & & \\
\hline & & $\begin{array}{c}\text { Non-group A or B Streptococcus } \\
\text { (I) }\end{array}$ & & & \\
\hline \multicolumn{6}{|c|}{ Independent test set I $(n=33)$} \\
\hline Number of subjects & 13 & II & 4 & 2 & 3 \\
\hline Mean age in years (range) & $50(18-70)$ & $56(37-70)$ & $50(39-64)$ & $49(48-50)$ & $38(35-43)$ \\
\hline Sex (male/female) & $11 / 2$ & $6 / 5$ & $3 / 1$ & $0 / 2$ & $0 / 3$ \\
\hline Survivors/non-survivors & $12 / 1$ & $6 / 5$ & & & \\
\hline \multirow[t]{6}{*}{ Organisms (n) } & B. pseudomallei (13) & Coagulase-negative staphylococci (6)* & & & \\
\hline & & E. coli $(\mathrm{I})$ & & & \\
\hline & & Enterococcus spp. (I) & & & \\
\hline & & S. aureus (I) & & & \\
\hline & & K. pneumoniae (I) & & & \\
\hline & & S. pneumoniae (I) & & & \\
\hline \multicolumn{6}{|c|}{ Independent test set $2(n=25)$} \\
\hline Number of subjects & 8 & 7 & & 5 & 5 \\
\hline Mean age in years (range) & $47(40-56)$ & $61(43-8 I)$ & & $57(50-7 \mathrm{I})$ & $44(37-67)$ \\
\hline Sex (male/female) & $4 / 4$ & $2 / 5$ & & $0 / 5$ & $3 / 2$ \\
\hline Survivors/non-survivors & $3 / 5$ & $5 / 2$ & & $5 / 0$ & $5 / 0$ \\
\hline \multirow[t]{6}{*}{ Organisms (n) } & B. pseudomallei (8) & A. hydrophila $(\mathrm{I})^{\dagger}$ & & & \\
\hline & & Corynebacterium spp. (I) & & & \\
\hline & & E. coli $(2)^{\dagger}$ & & & \\
\hline & & S. aureus (I) & & & \\
\hline & & Enterococcus spp. (I) & & & \\
\hline & & E. faecium $(\mathrm{I})$ & & & \\
\hline
\end{tabular}

\footnotetext{
*Three in six patients were positive in two sets of blood cultures. tPatients were positive in two sets of blood cultures.
}

and microbiological data are available in Table 2 and Additional data file 1 .

The first independent test set (test set 1) is composed of 33 samples: 24 patients with sepsis, including 13 patients with septicemic melioidosis, and 11 patients with sepsis and isolation of other organisms (6 coagulase-negative staphylococci, $1 S$. aureus, 1 Streptococcus pneumoniae, 1 Klebsiella pneumoniae, 1 Enterococcus spp., and 1 E. coli); and 9 control samples, including 4 patients who recovered from melioidosis, 2 patients with T2D, and 3 healthy donors from the same endemic area. Demographic, clinical and microbiological data are available in Table 3 and Additional data file 1.

The second independent test set (test set 2) is composed of 25 samples: 15 patients with sepsis, including 8 patients with septicemic melioidosis, and 7 patients with sepsis and isolation of other organisms ( 2 E. coli, $1 S$. aureus, 1 Corynebacterium spp., 1 Enterococcus spp., 1 Enterococcus faecium, and 1 Aeromonas hydrophila); and 10 control samples, including 5 patients with T2D and 5 healthy donors. The demographic, 


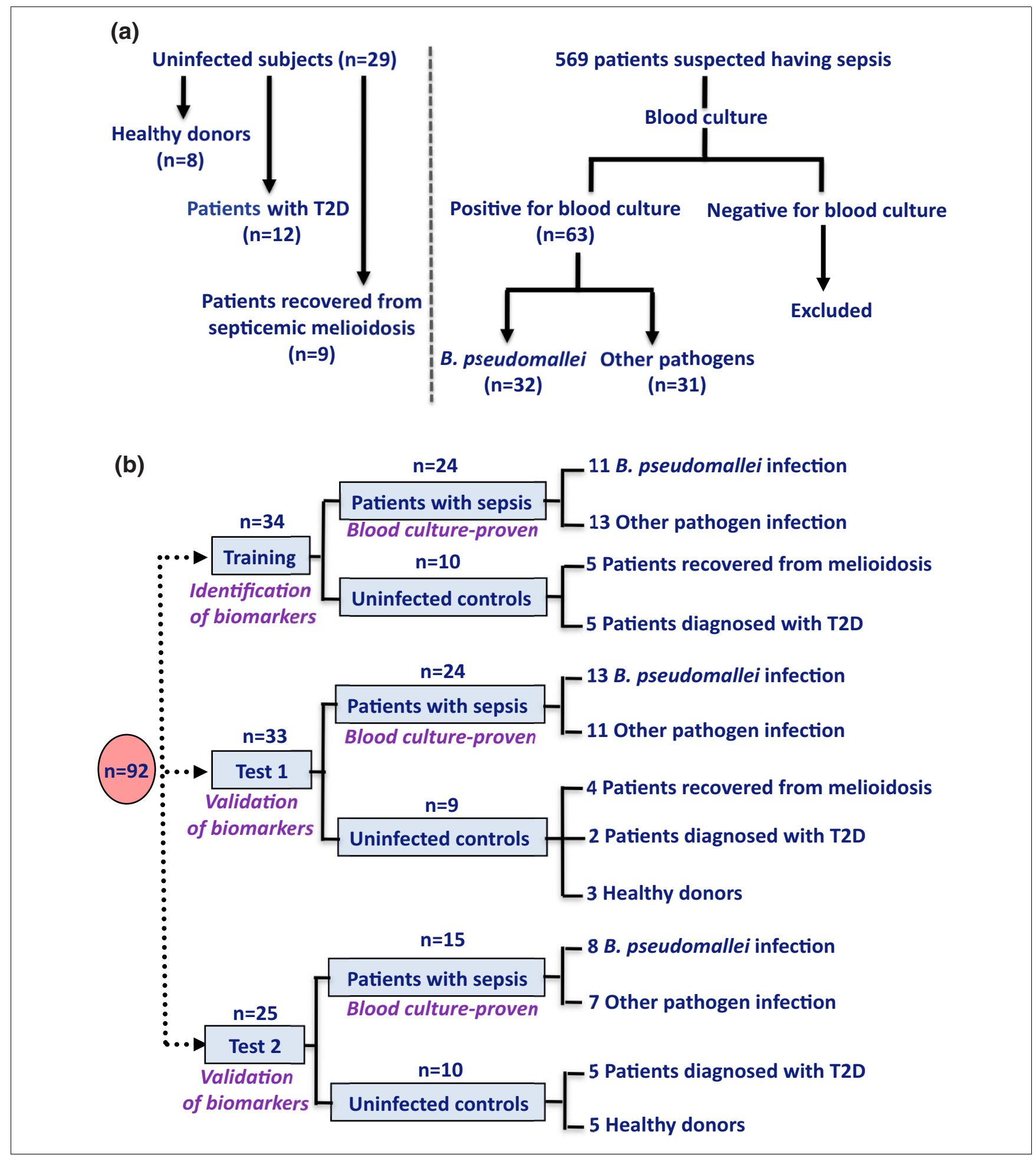

Figure I

Subject enrolment and study design. (a) Recruitment strategy. A total of 598 subjects consisting of 29 uninfected controls and 569 patients diagnosed with sepsis were recruited in this study. Of the patients diagnosed with sepsis (569 subjects), only those with positive blood cultures (63 subjects) were included for further study. Subjects who had no signs of infection (29 subjects) were also recruited to constitute an uninfected control group, including healthy donors, patients diagnosed with T2D, and patients who had recovered from melioidosis. Subjects for this latter group could not be recruited in our second validation set. (b) Study design. The diagram depicts the composition of the training and independent test sets. Of 92 subjects enrolled in this study, 34 were assigned to the training set, 33 were assigned to the test set I, and 25 were assigned to the test set 2 . T2D, type 2 diabetes. 
Table 2

\begin{tabular}{|c|c|c|c|c|c|c|}
\hline Sample ID & Age (years) & Sex & Bacterial isolation & $\begin{array}{l}\text { Antibiotherapy before } \\
\text { blood collection }\end{array}$ & Underlying diseases & Survival \\
\hline \multicolumn{7}{|c|}{ Other sepsis $(n=13)$} \\
\hline $100 I^{*}$ & 52 & Male & $\begin{array}{l}\text { Streptococcus non- } \\
\text { group } A \text { or } B\end{array}$ & Ceftriaxone & - & Non-survivor \\
\hline $1002 \dagger \neq$ & 52 & Female & A. baumannii & Ceftazidime, bactrim & T2D, CRF, lung edema & Survivor \\
\hline $1004 * \neq$ & 45 & Male & Salmonella serotype B & Cloxacillin, ceftriaxone & $\mathrm{T} 2 \mathrm{D}$, arthritis & Survivor \\
\hline $1006 * \S$ & 37 & Male & C. albicans & $\begin{array}{l}\text { Ceftriaxone, sulperazone, } \\
\text { bactrim }\end{array}$ & $\begin{array}{l}\text { HIV infection, } \\
\text { tuberculosis }\end{array}$ & Survivor \\
\hline $1007^{*}$ & 73 & Female & Corynebacterium spp. & - & $\begin{array}{l}\text { NSAID-induced GI } \\
\text { bleeding }\end{array}$ & Non-survivor \\
\hline $1008+\pi$ & 70 & Female & E. coli & Bactrim, ceftazidime & $\mathrm{T} 2 \mathrm{D}$ & Survivor \\
\hline $1009 *$ & 52 & Female & S. aureus & Ceftazidime, cloxacillin & T2D, knee abscess & Survivor \\
\hline $1010 t \neq \#$ & 72 & Female & E. coli & Ceftriaxone & T2D, CRF & Survivor \\
\hline $1011 * \pi$ & 38 & Female & E. coli & - & HCV infection & Survivor \\
\hline $1012 * \S$ & 69 & Female & C. albicans & Ceftazidime & RF & Survivor \\
\hline $1013^{*}$ & 74 & Female & Corynebacterium spp. & Ceftazidime, clarithromycin & $\begin{array}{l}\text { Chronic heart failure, } \\
\text { COPD }\end{array}$ & Survivor \\
\hline $1014 *$ & 54 & Female & Salmonella spp. & $\begin{array}{l}\text { Ceftriaxone, ceftazidime, } \\
\text { levofloxacin }\end{array}$ & $\begin{array}{l}\text { T2D, endometrial } \\
\text { cancer, ITP }\end{array}$ & Survivor \\
\hline $1015 * \S$ & 41 & Male & C. albicans & Ceftazidime & HIV infection & Survivor \\
\hline \multicolumn{7}{|c|}{$\begin{array}{l}\text { Septicemic } \\
\text { melioidosis }(n=1 I)\end{array}$} \\
\hline Mool* & 68 & Male & B. pseudomallei & Ceftazidime, bactrim & $\begin{array}{l}\text { Chronic heart failure, } \\
\text { COPD }\end{array}$ & Non-survivor \\
\hline M002* & 43 & Female & B. pseudomallei & Ceftriaxone, ceftazidime & T2D & Survivor \\
\hline M003* & 55 & Male & B. pseudomallei & Ceftazidime & - & Non-survivor \\
\hline M006* & 46 & Male & B. pseudomallei & Ceftriaxone & $\mathrm{T} 2 \mathrm{D}$, chirrosis & Non-survivor \\
\hline M007* & 50 & Male & B. pseudomallei & Ceftazidime, tazocin & Lung cancer & Survivor \\
\hline M008* & 70 & Female & B. pseudomallei & Ceftazidime, bactrim & T2D & Non-survivor \\
\hline M009* & 48 & Female & B. pseudomallei & Sulperazone & $\mathrm{T} 2 \mathrm{D}$ & Survivor \\
\hline MOIO* & 48 & Male & B. pseudomallei & $\begin{array}{l}\text { Ceftriaxone, ceftazidime, } \\
\text { doxycycline }\end{array}$ & T2D & Survivor \\
\hline $\mathrm{MOI} 2^{*}$ & 56 & Male & B. pseudomallei & $\begin{array}{l}\text { Sulperazone, bactrim, } \\
\text { cetazidime }\end{array}$ & TID, ARF & Survivor \\
\hline MOI4* & 65 & Female & B. pseudomallei & Cloxacilin, ceftazidime & T2D, chirrosis & Non-survivor \\
\hline MOI5* & 41 & Male & B. pseudomallei & Bactrim, ceftazidime & - & Survivor \\
\hline
\end{tabular}

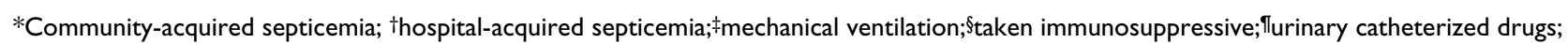
¥blood transfused. ARF, acute renal failure; COPD, chronic obstructive pulmonary disease; CRF, chronic renal failure; GI, gastrointestinal tract; NSAID, non-steroidal anti-inflammatory drug; RF, renal failure; T2D, type 2 diabetes; TP, idiopathic thrombocytopenic purpura.

clinical data and microbiological data are available in Table 4 and Additional data file 1.

All groups were similar in terms of race. There was no statistically significant difference in age among the data sets and disease status groups (ANOVA overall $\mathrm{F}$ test, $P$-value $=$ o.0884). There was also no statistically significant difference in gender among the data sets and disease groups (Fisher's exact test with Bonferroni correction, all $P$-values $\geq 0.274)$. No statistically significant differences were found between whole blood samples collected from patients with septicemic melio- idosis and patients with sepsis and isolation of other organisms in the training and the two test sets concerning the total leukocyte, platelet, neutrophil, lymphocyte, and monocyte blood cell counts (Table S1 in Additional data file 2). Out of 92 subjects, 58 were diagnosed with T2D (63\%), a well-documented risk factor for melioidosis. Of these 58 diabetic subjects, 17 were uninfected controls whereas 41 were septic patients. Pneumonia was found in 20 patients with melioidosis $(63 \%)$ and in 12 of the septic patients with infections caused by other organisms (39\%). In addition, 4 out of 63 patients with sepsis were immunocompromised, including 2 
Table 3

Characteristics of patients in the independent test set I

\begin{tabular}{|c|c|c|c|c|c|c|}
\hline Sample ID & Age (years) & Sex & Bacterial isolation & $\begin{array}{l}\text { Antibiotherapy before } \\
\text { blood collection }\end{array}$ & Underlying diseases & Survival \\
\hline \multicolumn{7}{|c|}{ Other sepsis $(n=I I)$} \\
\hline $1016^{*}+$ & 61 & Female & $\begin{array}{l}\text { Coagulase-negative } \\
\text { staphylococci }\end{array}$ & $\begin{array}{l}\text { Ceftazidime, bactrim, } \\
\text { Sulperazole }\end{array}$ & Hematemesis & Survivor \\
\hline $1017^{*} \ddagger \S$ & 50 & Male & $\begin{array}{l}\text { Coagulase-negative } \\
\text { staphylococci }\end{array}$ & $\begin{array}{l}\text { Ceftriaxone, ceftazidime, } \\
\text { doxycycline, cloxacillin }\end{array}$ & $\begin{array}{l}\text { Acute pancreatitis, } \\
\text { nephrotic syndrome }\end{array}$ & Survivor \\
\hline $1018 \$ 7 \%$ & 57 & Male & $\begin{array}{l}\text { Coagulase-negative } \\
\text { staphylococci\# }\end{array}$ & Vancomycin & T2D, CRF & Survivor \\
\hline $1019^{a}$ & 58 & Female & Staphylococcus aureus & Cloxacillin, ceftazidime & T2D, wound & Survivor \\
\hline 1020平 & 66 & Female & $\begin{array}{l}\text { Coagulase-negative } \\
\text { staphylococci\# }\end{array}$ & Ceftazidime, ceftriaxone & T2D, ARF, tuberculosis & Non-survivor \\
\hline $1021 \pi$ & 54 & Female & Enterococcus spp. & Ceftazidime, cloxacilin & T2D, abscess & Non-survivor \\
\hline 1022\$ा & 37 & Male & $\begin{array}{l}\text { Coagulase-negative } \\
\text { staphylococci\# }\end{array}$ & Ceftriaxone, ceftazidime & T2D, ARF & Non-survivor \\
\hline $1023 \pi$ & 70 & Female & E. coli & Doxycycline, ceftazidime & $\mathrm{T} 2 \mathrm{D}$ & Non-survivor \\
\hline $1024 \pi ¥$ & 56 & Male & $\begin{array}{l}\text { Coagulase-negative } \\
\text { staphylococci }\end{array}$ & Meropenem, ceftazidime & T2D, RF & Survivor \\
\hline $1025 *$ & 50 & Male & S. pneumoniae & Ceftriaxone, meropenem & $\mathrm{T} 2 \mathrm{D}$ & Non-survivor \\
\hline $1026 \pi$ & 57 & Male & K. pneumoniae & $\begin{array}{l}\text { Ceftriaxone, ceftazidime, } \\
\text { bactrim }\end{array}$ & $\mathrm{T} 2 \mathrm{D}$ & Survivor \\
\hline \multicolumn{7}{|c|}{$\begin{array}{l}\text { Septicemic } \\
\text { melioidosis }(n=13)\end{array}$} \\
\hline M016\% & 39 & Male & B. pseudomallei & $\begin{array}{l}\text { Ceftazidime, bactrim, } \\
\text { doxycycline }\end{array}$ & $\mathrm{T} 2 \mathrm{D}$ & Survivor \\
\hline MoI7T & 52 & Female & B. pseudomallei & Norfloxacin, ceftazolin & $\mathrm{T} 2 \mathrm{D}$ & Survivor \\
\hline M020ा & 61 & Male & B. pseudomallei & $\begin{array}{l}\text { Ceftriaxone, doxycycline, } \\
\text { ceftazidime }\end{array}$ & - & Survivor \\
\hline M02। & 56 & Female & B. pseudomallei & Ceftriaxone, ceftazidime & $\mathrm{T} 2 \mathrm{D}$ & Survivor \\
\hline M022 & 18 & Male & B. pseudomallei & Ceftazidime, cactrim & $\mathrm{T} 2 \mathrm{D}$ & Survivor \\
\hline M023T & 63 & Male & B. pseudomallei & Bactrim, ceftazidime & $\mathrm{T} 2 \mathrm{D}$ & Survivor \\
\hline M024 & 44 & Male & B. pseudomallei & Meropenem & $\mathrm{T} 2 \mathrm{D}, \mathrm{RF}$ & Survivor \\
\hline M025ा & 57 & Male & B. pseudomallei & Ceftazidime & $\mathrm{T} 2 \mathrm{D}$ & Survivor \\
\hline M026ा & 48 & Male & B. pseudomallei & $\begin{array}{l}\text { Ceftazidime, doxycycline, } \\
\text { bactrim }\end{array}$ & $\mathrm{T} 2 \mathrm{D}$ & Survivor \\
\hline M027爪 & 44 & Male & B. pseudomallei & $\begin{array}{l}\text { Ceftriaxone, ceftazidime, } \\
\text { meropenem }\end{array}$ & ARF & Survivor \\
\hline M028T & 70 & Male & B. pseudomallei & $\begin{array}{l}\text { Ceftazidime, levofloxacin, } \\
\text { bactrim }\end{array}$ & $\mathrm{T} 2 \mathrm{D}$ & Survivor \\
\hline M029ा & 50 & Male & B. pseudomallei & Ceftriaxone, ceftazidime & CRF & Non-survivor \\
\hline M030ा & 44 & Male & B. pseudomallei & Ceftazidime, ceftriazone & $\mathrm{T} 2 \mathrm{D}$, tuberculosis & Survivor \\
\hline
\end{tabular}

*Hospital-acquired septicemia; †long hospitalization; ¥taken immunosuppressive drugs; §dialysis; ๆcommunity-acquired septicemia; $¥$ mechanical ventilation; " wounds. \#Positive by two sets of blood cultures. ARF, acute renal failure; CRF, chronic renal failure; RF, renal failure; T2D, type 2 diabetes.

patients under immunosuppressive therapy and 2 patients with underlying HIV infection.

\section{Blood transcriptional profiles of septic patients and healthy or diabetic controls are distinct}

We first wanted to determine whether transcriptional profiles of septicemic patients were distinct from those of healthy individuals and individuals with T2D. We started by carrying out unsupervised analyses that consist in exploring molecular signatures in a dataset without a priori knowledge of sample phenotype or grouping. Blood profiles from the training dataset (24 septicemic patients and 10 controls) were first subjected to this analysis. Filters were applied to remove transcripts that are not detected in at least $10 \%$ of all samples (detection $P$-value $<0.01$ ), and that are expressed at similar levels across all conditions, that is, present little deviation 
Table 4

Characteristics of patients in the independent test set 2

\begin{tabular}{|c|c|c|c|c|c|c|}
\hline Sample ID & Age (years) & Sex & Bacterial isolation & $\begin{array}{l}\text { Antibiotherapy before } \\
\text { blood collection }\end{array}$ & Underlying diseases & Survival \\
\hline \multicolumn{7}{|c|}{ Other sepsis $(n=7)$} \\
\hline $1027^{*}$ & 64 & Female & E. colit & Fortum, ceftriaxone & UGIB & Non-survivor \\
\hline $1028 \ddagger$ & 81 & Female & Corynebacterium spp. & $\begin{array}{l}\text { Ceftriaxone, fortum, } \\
\text { clindamycin }\end{array}$ & $\mathrm{T} 2 \mathrm{D}$ & Survivor \\
\hline $1029 \ddagger$ & 74 & Female & S. aureus & $\begin{array}{l}\text { Fortum, ceftriaxone, } \\
\text { tazocin }\end{array}$ & $\begin{array}{l}\text { Asthma, emphysema, } \\
\text { ARF }\end{array}$ & Survivor \\
\hline $103 \mid *$ & 48 & Male & Enterococcus spp. & Fortum & Urinary tract infection & Survivor \\
\hline $1032 *$ & 54 & Female & E. faecium & Fortum, tazocin & $\mathrm{T} 2 \mathrm{D}$, respiratory failure & Non-survivor \\
\hline 1033* & 63 & Female & E. colit & $\begin{array}{l}\text { Tazocin, ceftriaxone, } \\
\text { fortum }\end{array}$ & $\mathrm{T} 2 \mathrm{D}$, ovarian cancer & Survivor \\
\hline $1034 *$ & 43 & Male & A. hydrophila & Tazocin & - & Survivor \\
\hline \multicolumn{7}{|c|}{$\begin{array}{l}\text { Septicemic melioidosis } \\
(n=8)\end{array}$} \\
\hline M03I* & 49 & Male & B. pseudomallei & Fortum, bactrim, tazocin & $\mathrm{T} 2 \mathrm{D}$ & Non-survivor \\
\hline M032* & 54 & Male & B. pseudomallei & $\begin{array}{l}\text { Fortum, doxycycline, } \\
\text { sulperazone }\end{array}$ & $\mathrm{T} 2 \mathrm{D}$ & Non-survivor \\
\hline M033* & 44 & Male & B. pseudomallei & $\begin{array}{l}\text { Fortum, sulperazone, } \\
\text { bactrim, ciprofloxacin }\end{array}$ & $\mathrm{T} 2 \mathrm{D}$ & Survivor \\
\hline M034* & 40 & Female & B. pseudomallei & $\begin{array}{l}\text { Fortum, bactrim, } \\
\text { ceftazidime, ceftriaxone }\end{array}$ & $\mathrm{T} 2 \mathrm{D}$ & Survivor \\
\hline M035* & 56 & Male & B. pseudomallei & $\begin{array}{l}\text { Ceftriaxone, ceftazidime, } \\
\text { fortum }\end{array}$ & COPD, T2D & Non-survivor \\
\hline M036* & 41 & Female & B. pseudomallei & Ceftriaxone, ceftazidime & $\mathrm{T} 2 \mathrm{D}$ & Non-survivor \\
\hline M037* & 42 & Female & B. pseudomallei & Bactrim, fortum, cloxacillin & $\mathrm{T} 2 \mathrm{D}$ & Survivor \\
\hline M038* & 49 & Female & B. pseudomallei & $\begin{array}{l}\text { Ceftriaxone, fortum, } \\
\text { ceftazidime, levofloxacin }\end{array}$ & - & Non-survivor \\
\hline
\end{tabular}

*Community-acquired septicemia; †hospital-acquired septicemia. †Positive by two sets of blood cultures. ARF, acute renal failure; COPD, chronic obstructive pulmonary disease; T2D, type 2 diabetes; UGIB, upper gastrointestinal bleeding.

from the median intensity value calculated across all samples (less than 2-fold and 200 intensity units from the median; see Materials and method section for details). From a total of 48,701 probes arrayed on the Illumina Hu6 V2 beadchip, 16,400 transcripts passed the detection filter and 2,785 transcripts passed both filters.

This set of 2,785 transcripts was used in an unsupervised hierarchical clustering analysis where transcripts are ordered horizontally and samples (conditions) vertically, according to similarities in expression patterns (Figure 2a). The resulting heatmap reveals the molecular heterogeneity of this sample set. The molecular classification obtained through hierarchical clustering is then compared with phenotypic classification of the samples: out of the ten uninfected controls, nine samples were clustered together on a branch of the condition tree (region R1) that is distinct from that of septicemic patients (regions R2, R4, and R5). One outlying uninfected control clustered together with septicemic patients (sample Roo1 in region $\mathrm{R}_{3}$ ). The expression pattern for this outlying sample appeared nonetheless distinct from that of septicemia and it was excluded from subsequent class comparison analyses.

We further explored the molecular heterogeneity of this sample set through principal component analysis (PCA; Figure S1 in Additional data file 2). PCA is a useful tool to reduce the dimension and complexity of microarray data. The 2,785 most variable transcripts selected above were decomposed into 7 principal components (PCs). The first 3 major PCs accounted for $40.1 \%$ (PC1), $18.2 \%$ (PC2), and 6.2\% (PC3) of the variability observed for these conditions. This threedimensional plot confirmed the segregation of uninfected controls from septicemic patients with the exception of the same outlying sample (sample Roo1).

We repeated this analysis for the independent test set 1 ( $\mathrm{n}=$ 33) using the same 2,785 transcripts previously identified in the analysis of the training set. Once again, unsupervised hierarchical clustering revealed distinctive transcriptional profiles separating uninfected controls (region R6) from patients with sepsis (regions R8, R9, and R10) (Figure 2b). 
TRAINING

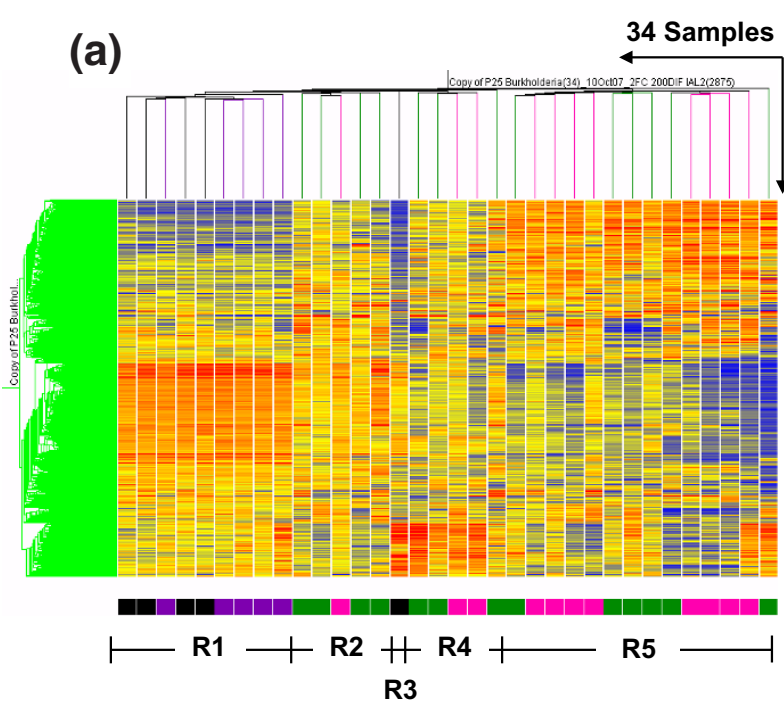

Controls recovered from melioidosis

$\square$ Controls diagnosed with T2D
TEST 1

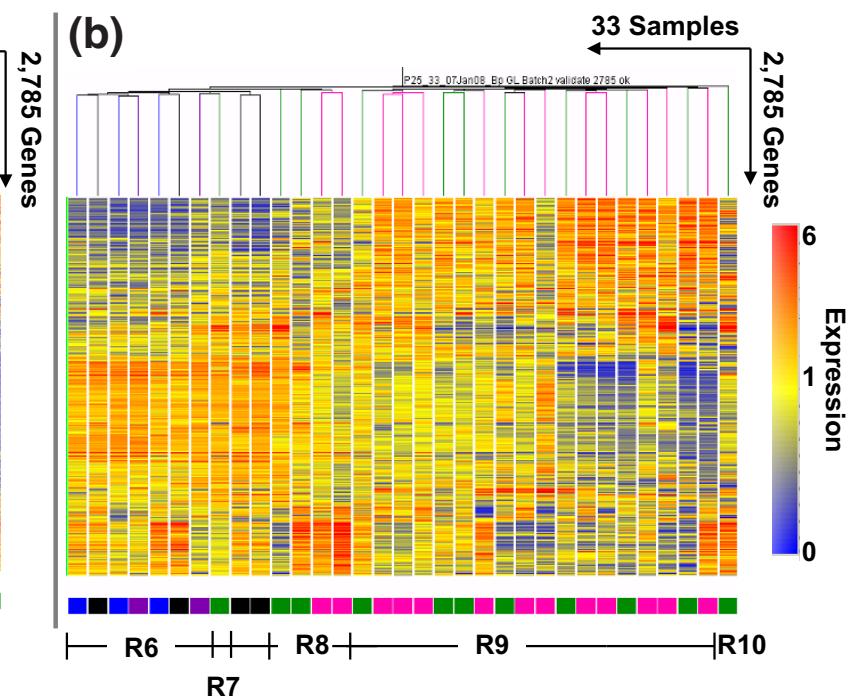

$\square$ Patients infected by other organisms

\section{Patients with melioidosis}

$\square$ Healthy donors

Figure 2

Unsupervised hierarchical clustering of blood transcriptional profiles of septic patients. Transcripts with 2-fold over- or under-expression compared with the median of all samples and differential expression values greater than 200 from the median for each gene in at least 2 samples in the training set were selected for unsupervised analysis ( $n=2,785$ transcripts). (a) A heatmap resulting from hierarchical clustering of transcripts and conditions (subjects) was generated for the training set. (b) The same gene tree of these 2,785 transcripts was then used to generate a heatmap for the first independent test set (test set I) using hierarchical clustering of conditions as before. The color conventions for heatmaps are as follows: red indicates over-expressed transcripts; blue represents underexpressed transcripts; and yellow indicates transcripts that do not deviate from the median. Study group is marked as follows: patients with melioidosis are indicated by pink rectangles; patients with sepsis due to other organisms by green rectangles; uninfected controls who recovered from melioidosis by black rectangles; T2D patients by purple rectangles; and healthy donors by blue rectangles. This unsupervised hierarchical clustering of blood transcriptional profiles was observed to segregate into five distinct regions in both training (regions RI to R5) and test sets (regions R6 to RI0).

Thus, the results of the unsupervised analysis clearly established the existence of a robust blood transcriptional signature in the context of sepsis that is distinct from that of uninfected controls. Indeed, the sample grouping (separation of healthy controls and T2D compared to sepsis) and lack thereof (non-separation of healthy controls compared to T2D) observed following unsupervised hierarchical clustering (Figure 2) and PCA (Figure S1 in Additional data file 2) indicates that the transcriptional profile of T2D patients is more similar to healthy controls than to patients with sepsis. This suggests that the transcriptional perturbation induced by melioidosis or sepsis is of such a magnitude as to render any such effect from T2D undetectable in comparison.

To examine the biological significance of the 2,785 transcript signature, we extracted annotations from the Database for Annotation, Visualization and Integrated Discovery (DAVID) using Expression Analysis Systematic Explorer (EASE). The major biological Gene Ontology term enrichments categorized from these 2,785 transcripts are shown in Figure S2 in Additional data file 2. This analysis associated transcripts with several biological categories, including defense response (CD55, CD59, LTF, TLR2), immune system process (GBP6, $H L A-A, H L A-D M A, B C L 2)$, response to stress (ZAK, GP9, $D U S P 1, P T G S 1)$, and inflammatory response (CFH, TLR4, IL1B, SERPING1) [26].

Next, we identified and independently validated sets of transcripts differentially expressed between uninfected controls and patients with sepsis by carrying out direct comparison between these two groups (supervised analysis). Starting from the list of genes present in at least $10 \%$ of samples defined above ( $n=16,400)$, we performed statistical comparisons (Welch $t$-test, $P<0.01$ ) with three different stringencies 
of multiple testing corrections and returned sets of transcripts for which expression levels were significantly different between the two study groups (Table S2 and Figure $\mathrm{S}_{3}$ in Additional data file 2). Using the most stringent Bonferroni correction for controlling type I error, 2,733 transcripts were found differentially expressed between these two groups. Applying a more liberal correction, the Benjamini and Hochberg false discovery rate, to the analysis yielded an expanded list of 7,377 transcripts differentially expressed between these two groups (false discovery rate $=1 \%$ ). Finally, performing the statistical analysis without any multiple testing correction yielded 8,096 differentially expressed transcripts with 164 transcripts expected to be positive by chance alone. These 3 transcriptional signatures identified using different statistical stringencies were then validated independently in the first test set composed of 9 uninfected controls and 24 patients with sepsis. We found that hierarchical clustering discriminated perfectly between the two groups in this independent test set when using the probes identified with the Bonferroni correction (Figure $S_{3} f$ in Additional data file 2). Class prediction analysis further confirmed these results since a set of 10 predictors gave over 95\% in sensitivity and specificity in the training set (K-nearest neighbors; leave-one-out cross-validation) and $96 \%$ sensitivity and $89 \%$ specificity in the first independent test set (Table $\mathrm{S}_{3}$ in Additional data file 2).

In conclusion, these results demonstrate that whole blood transcriptional profiles in patients with sepsis and in noninfected controls are distinct.

\section{Blood transcriptional profiles of septic patients are heterogeneous}

While the signature of sepsis is clearly distinct from that of uninfected controls, unsupervised analyses revealed that it was also heterogeneous. Indeed, distinct patterns are discernable on the heatmaps generated from the training set (Figure 2a, regions $\mathrm{R}_{2}, \mathrm{R}_{4}$, and $\mathrm{R}_{5}$ ) and test set 1 (Figure $2 \mathrm{~b}$, regions R8, R9, and R10). This heterogeneity cannot be explained by etiological differences since the pathogen species identified are distributed among the different regions (R2: 2 C. albicans, 1 A. baumannii, 1 Corynebacterium spp., and 1 B. pseudomallei; R4: 1 Corynebacterium spp., 1 Salmonella serotype B, 1 E. coli, and 2 B. pseudomallei; R5: 1 Salmonella spp., $1 S$. aureus, 1 Streptococcus non group A or B, 1 C. albicans, 2 E. coli, and 8 B. pseudomallei; R8: 2 coagulase-negative staphylococci, 2 B. pseudomallei; R9: 4 coagulase-negative staphylococci, 1 S. pneumoniae, $1 \mathrm{E}$. coli, $1 \mathrm{~K}$. pneumoniae, $11 \mathrm{~B}$. pseudomallei; R10: 1 Enterococcus spp.), nor can it be attributed to differences in treatment, co-morbidity or pulmonary involvement (Figure 3a, b).

A metric that we have developed to quantify global transcriptional changes over a pre-determined baseline was used to further investigate the source of heterogeneity in the sepsis patient signature (molecular distance; see Materials and methods for details). Cumulative distances from the unin- fected control baseline increased progressively from region $\mathrm{R} 2$ to regions $\mathrm{R} 4$ and $\mathrm{R} 5$ of the training set (Figure $4 \mathrm{a}$ ), and from region $\mathrm{R} 6$ to regions $\mathrm{R} 8, \mathrm{R} 9$ and $\mathrm{R} 10$ of the test set 1 (Figure 4b). As indicated on the same graphs we also observed that most fatalities occurred in patients found in regions $\mathrm{R}_{5}$ and $\mathrm{R} 9$. Septic patients who died showed multiple organ dysfunction when compared to those who survived (Figure 3a, b). The number of patients with severe sepsis was higher in region $\mathrm{R} 5$ compared to regions $\mathrm{R} 2$ and $\mathrm{R} 4$ (86\%, 40\%, and 40\%, respectively; Figure 4a). Most patients with pneumonia, whether due to melioidosis or other organisms, were also in R5 (Figure 3a). Similarly, the number of patients with severe sepsis increased from region $\mathrm{R} 8$ (25\%) to $\mathrm{R} 9$ (67\%) in test set 1 (Figure $4 \mathrm{~b}$ ). Despite all patient samples being obtained within 48 hours of the diagnosis of sepsis, these results suggest that the heterogeneity of the blood transcriptional profiles observed among patients with sepsis may be linked to differences in degrees of disease severity.

\section{Blood transcriptional profiles of septic patients are heterogeneous}

Recently, our group has developed a transcriptional modulebased analysis that provides pre-determined annotations through literature profiling of sets of functionally related transcripts [27]. This data dimension reduction approach groups transcripts according to similarities in expression pattern in the blood of patients across a wide range of diseases. Focusing the analysis on sets of coordinately expressed transcripts facilitates functional interpretation of the data, with the activity of annotated modules mapped on a standardized grid format. Furthermore, this approach proved robust in comparisons carried out across different microarray platforms [28].

To facilitate the biological interpretation of the distinct sepsis signatures identified in the present study, we applied this modular analysis strategy. Briefly, differences in expression levels between uninfected controls (region R1) and septic patients (regions R2, R4 or R5) for sets of coordinately expressed transcripts (that is, modules) are displayed on a grid (Figure 5). Each position on the grid is assigned to a given module; a red spot indicates an increase in expression level and a blue spot a decrease. The spot intensity is determined by the proportion of transcripts reaching significance for a given module ( $\geq \mathbf{2 0} \%$ of transcripts in a given module differentially expressed compared to the non-infected group, Mann-Whitney U-test $P<0.01)$. A posteriori biological interpretation by unbiased literature profiling has linked several modules to immune cells or pathways as indicated by a color code on the figure legend [27]. The modular map thus constructed for region R2 shows modest over-expression of interferon-inducible transcripts (M3.1: STAT1, IFI35, GBP1) and under-expression of transcripts linked to B-cells (M1.3: $E B F$, $B L N K, C D 72)$, ribosomal proteins (M2.4: ZNF32, $P E B P 1$, RPL36), or T-cells (M2.8: CD96, CD5, LY9) (Figure 5a). An increase in the number of altered modules and spot intensi- 


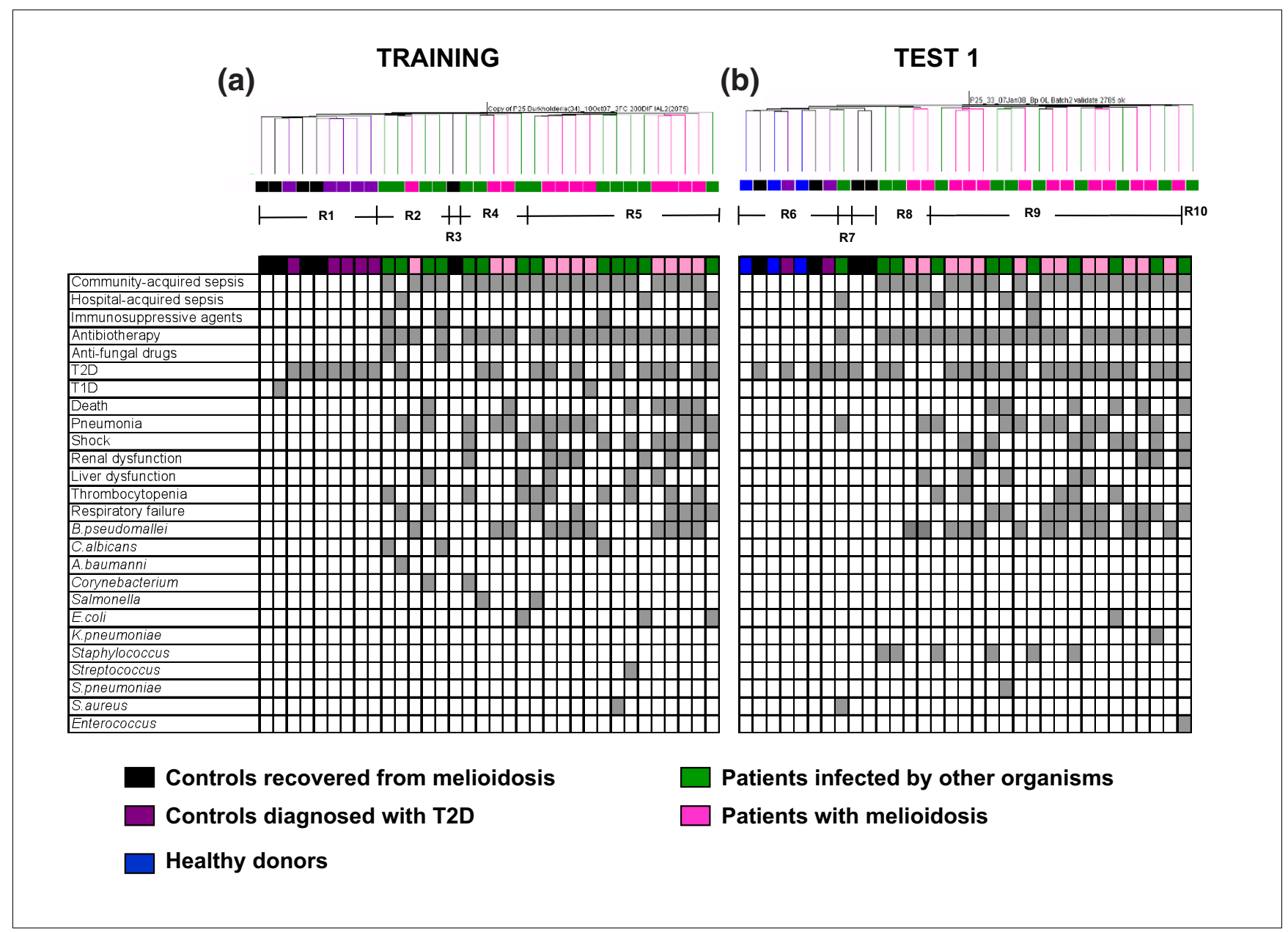

Figure 3

Comparison of phenotypic and clinical information with unsupervised condition clustering. The distribution of subjects who were defined as communityacquired or nosocomial septicemia, given antibiotics before blood collection (Antibiotherapy), diagnosed with TID or T2D, organ dysfunction, pneumonia, and microbial diagnosis is indicated on a grid aligned against the hierarchical condition tree generated through unsupervised clustering (Figure 2) for both (a) training and (b) test set I.

ties was observed when comparing region R4 to the uninfected control region (R1), thereby confirming the increased level of perturbation quantified through the earlier computation of cumulative distances (Figure 4). A pronounced overexpression of transcripts associated with neutrophils (M2.2: BPI, DEFA4, CEACAM8), myeloid lineage cells (M2.6: PA1L2, FCER1G, SIPA1L2), and erythrocytes (M2.3: ERAF, EPB49, MXI1) was observed, together with the under-expression of modules associated with ribosomal proteins (M2.4), T-cells (M2.8), and cytotoxic cells (M2.1: CD8B1, CD16o, $G Z M K)$. This set of modules was similarly affected in septic patients belonging to $\mathrm{R}_{5}$, but this time modules composed of interferon-inducible genes (M3.1: IFITM1, PLAC8, IFI35) and genes related to inflammation (M3.2: ICAM1, STX11, BCL3; M3.3: ASAH1, TDRD9, SERPINB1) were also overexpressed. Modular mapping carried out in turn for our first test set revealed a fingerprint for R9 that was most similar to $\mathrm{R} 5$, with both interferon and inflammation-related modules turned on. As described above, we observed that grouping of samples in regions $\mathrm{R}_{5}$ and $\mathrm{R} 9$ appeared to correlate with severity of septic illness. Increased abundance of transcripts associated with innate immune responses, including neutrophils, interferon, inflammation, and myeloid lineage, together with under expression of transcripts related to Tcells, B-cells, and cytotoxic cells, indicated substantial dysregulation of the host immune system in response to infection in those patients. This finding is in line with a recent report that found over-expression of transcripts corresponding to inflammation and innate immunity in the blood of patients with sepsis, while the abundance of transcripts related to adaptive immunity was decreased [29]. An interactive version of the module maps shown in Figure 5 is available online [30].

Neutrophils play a pivotal role in the defense against infections. In the present study, over-expression of genes related to this cell type (module M2.2) was observed in septic 
(a)

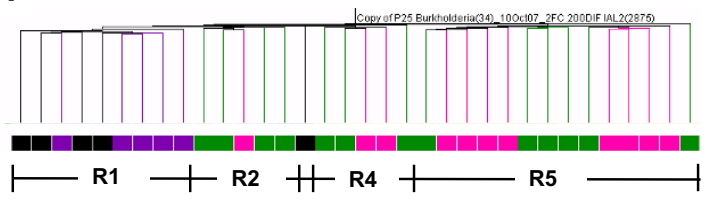

R3

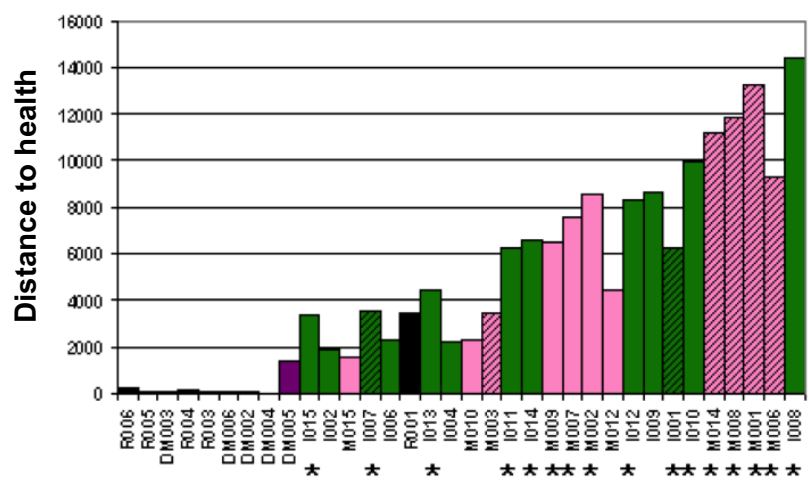

Controls recovered from melioidosis

Controls diagnosed with T2D

Healthy donors
TRAINING

(b)
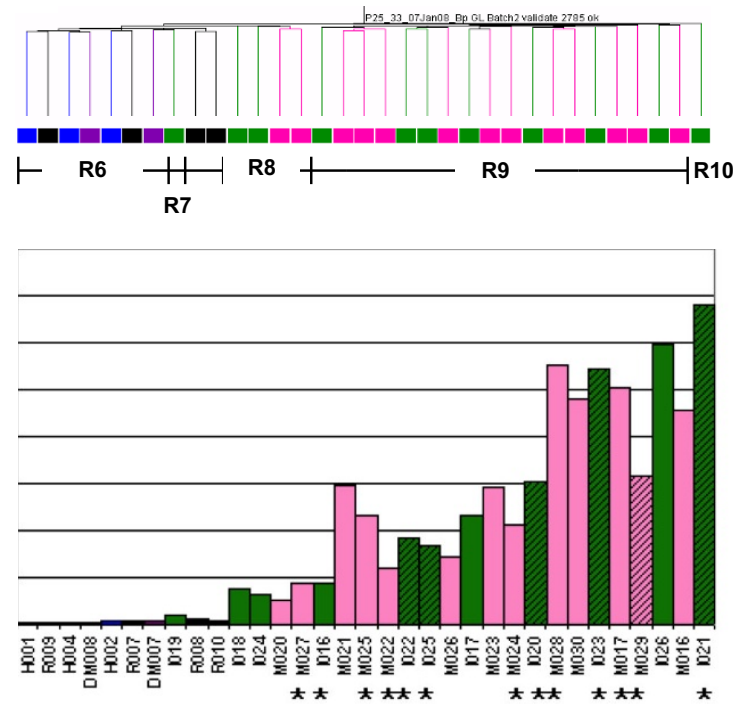

Patients infected by other organisms

Patients with melioidosis

Died from sepsis

* Severe sepsis

\section{Figure 4}

Comparison of molecular distances from baseline samples with unsupervised condition clustering. The list of 2,785 transcripts identified in the unsupervised analysis (Figure 2) was used to compute the 'molecular distance' between samples from patients with sepsis and uninfected control samples. (a, b) Region RI for the training set (a) and R6 for the first test set (b) were used as the baseline uninfected controls for all comparisons. Molecular distances for individual subjects are indicated on a histogram that is aligned against the hierarchical condition tree generated through unsupervised clustering (Figure 2). Study group is marked as follows: patients with melioidosis are indicated by pink rectangles; patients with sepsis due to other organisms by green rectangles; uninfected controls who recovered from melioidosis by black rectangles; T2D patients by purple rectangles; and healthy donors by blue rectangles. Patients who died from sepsis are indicated by diagonal shading within the bars. Patients with severe sepsis are indicated by asterisks.

patients compared to uninfected controls (Figure $\mathrm{S} 4$ in Additional data file 2). Increase in transcript abundance for genes included in this module may be an indication of an increase in the abundance of immature neutrophils (for example, $D E F A 1, D E F A 3, F A L L-39$ ) as was reported earlier in patients with systemic lupus erythematosus [27,31]. In particular, genes encoding neutrophil cell surface markers, such as ITGAM (CD11b), FCGR1 (CD64), CD62L, and CSF3R, were also over-expressed in septic patients and may be indicative of the activation status of neutrophils.

On the basis of the increased transcriptional perturbation seen in the blood of patients with severe sepsis (regions R4, R5, R9), as shown by both molecular cumulative distance and modular mapping analyses, we interpret the heterogeneity of the sepsis signatures as resulting from differences in levels of disease severity rather than differences in etiology. Longitudinal studies will have to be carried out in order to definitively address this point. We have in addition identified qualitative differences among the transcriptional fingerprints of patients with sepsis corresponding to distinct molecular phenotypes.

\section{Discovery and validation of a candidate biomarker signature for the diagnosis of septicemic melioidosis} We focused our biomarker discovery efforts on the prototypical signatures of sepsis established in both training and test sets. Samples clustering in $\mathrm{R} 5$ were used for the discovery of a diagnostic signature that distinguishes sepsis caused by $B$. pseudomallei from sepsis caused by other organisms. Class prediction identified a set of 37 classifiers that separated sam- 
(a)
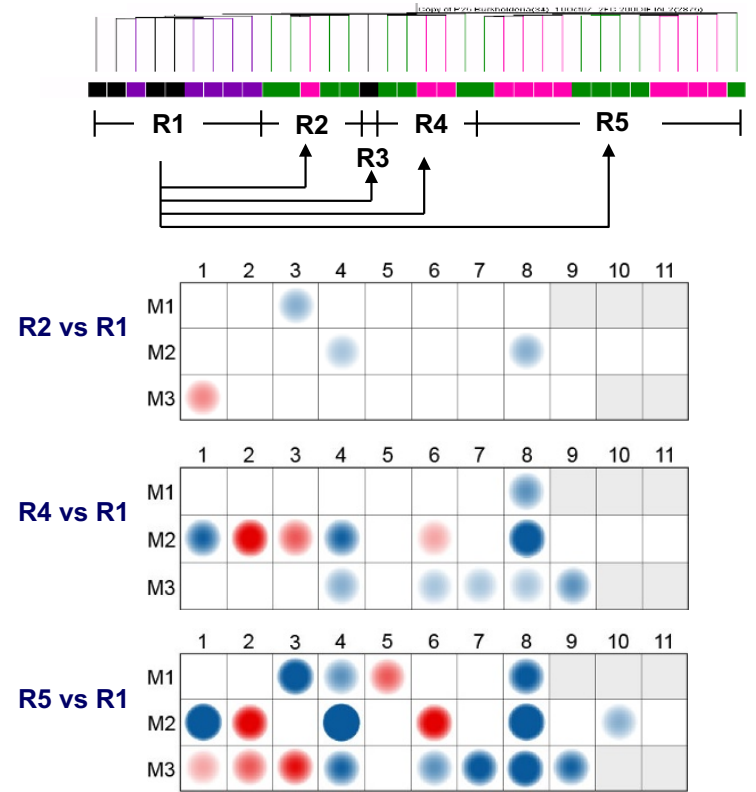

(b)
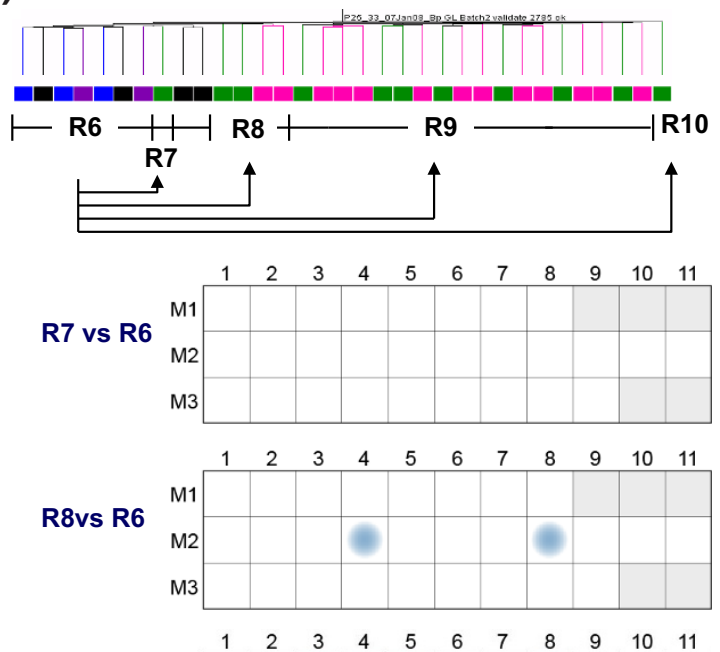

R9 vs R6

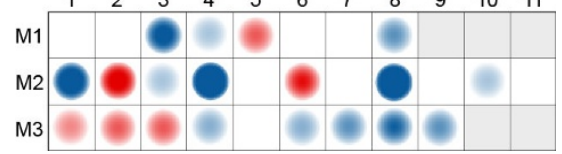

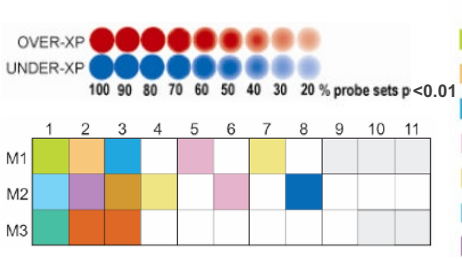

Functional Interpretation Plasma cells Platelets

B-cells

Myeloid Lineage

Ribosomal proteins

Cytotoxic cells

Neutrophils
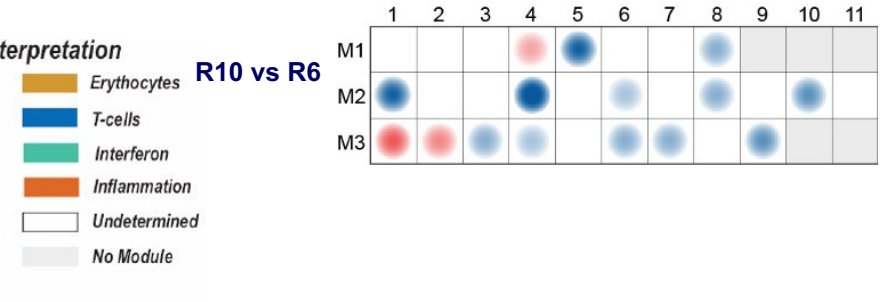

Figure 5

Modular transcriptional fingerprints for regions defined by unsupervised condition clustering. A modular analysis framework was used to generate modular transcriptional fingerprints for the major regions identified in Figure 2. Significant differences in expression levels in comparison to a baseline sample are indicated by a spot, with the intensity of the spot representing the proportion of significantly differentially expressed transcripts for each one of the transcriptional modules. The color of the spot indicates the direction of change of expression: red $=$ overexpressed, blue $=$ underexpressed. For the training set, region RI was used as the baseline for all comparisons, while for the first test set region R6 was used as the baseline. Functional interpretations are indicated by the color coded grid at the bottom left of the figure.

ples from the training set $\left(\mathrm{R}_{5} ; \mathrm{n}=14\right)$ with $100 \%$ accuracy in a leave-one-out cross-validation scheme (Figure 6a; K-nearest neighbors at cutoff $P$-value ratio $=0.9$ and number of neighbors $=5$ ). Next, the performance of this set of 37 candidate markers was evaluated independently. Samples from region $\mathrm{R} 9(\mathrm{n}=18)$ were classified with $78 \%$ accuracy $(82 \%$ sensitivity and $71 \%$ specificity; Figure $6 \mathrm{~b}$; K-nearest neighbors), with two melioidosis samples and two samples from patients with other infection being incorrectly classified (Table S4 in Additional data file 2). The transcripts forming this candidate biomarker signature are listed in Table 5, with 33 transcripts found to be over-expressed in patients with septicemic melioidosis and 4 underexpressed (IQWD1, OLR1, $A G P A T 9$, and $Z N F 281$ ). Antigen processing and presentation is the strongest functional association identified for this set of
37 classifiers $\left(P=1 \times 10^{-11}\right.$, Fischer's exact test; Figure $\left.7 \mathrm{a}\right)$. Some of the transcripts encode antigen processing and presentation (PSMB8, CD74) via major histocompatibility complex (MHC) class II molecules (HLA-DMA, HLA-DMB, HLA$D R A, H L A-D R B 2$, and HLA-DPA1), and the proteasome complex in the ubiquitin-proteasome system (UBE2L3, PSME2, $P S M B 2$, and $P S M B 5$ ) (Figure 7b). Some of the remaining transcripts are involved in proteolysis $\left(L A P_{3}, C F H\right.$, and $O L R 1$ ), the inflammatory response ( $A P O L 3$ and $A I F 1$ ), apoptosis and programmed cell death (SEPT4, ELMO2, and ZAK), cellular metabolic processes (ZAK, ZNF281, SSB, WARS, MSRB2, MTHFD2, DUSP3, and ASPHD2), or protein transport (STX11). RARRES3 is involved in negative regulation of cellular process, $L G A L S_{3} B P$ is related to the immune response, and $M A P B P I P$ is associated with the activation of 
Table 5

The 37 classifiers discriminated sepsis caused by B. pseudomallei from those by other organisms

\begin{tabular}{|c|c|c|c|}
\hline Rank & Abbreviation & Gene name & Gene accession \\
\hline I & FAM26F & Homo sapiens family with sequence similarity 26 , member $F$ & [GenBank:NM 001010919.1] \\
\hline 2 & MYOF & Myoferlin, transcript variant 2 & [GenBank:NM 133337.2] \\
\hline 3 & LAP3 & Leucine aminopeptidase 3 & [GenBank:NM 0I5907.2] \\
\hline 4 & HLA-DMA & Major histocompatibility complex, class II, DM alpha & [GenBank:NM 006/20.3] \\
\hline 5 & WARS & Tryptophanyl-tRNAsynthetase (WRS) & [GenBank:M617|5.I] \\
\hline 6 & RARRES3 & Retinoic acid receptor responder (tazarotene induced) 3 & [GenBank:NM 004585.3] \\
\hline 7 & HLA-DMB & Major histocompatibility complex, class II, DM beta & [GenBank:NM 002118.3] \\
\hline 8 & PSME2 & Proteasome (prosome, macropain) activator subunit 2 (PA28 beta) & [GenBank:NM 002818.2] \\
\hline 9 & Cl9orfl 2 & Chromosome 19 open reading frame 12, transcript variant 2 & [GenBank:NM 03।448.3] \\
\hline 10 & HLA-DRA & Major histocompatibility complex, class II, DR alpha & [GenBank:NM 019111.3] \\
\hline 11 & CD74 & $\begin{array}{l}\text { CD74 molecule, major histocompatibility complex, class II invariant chain transcript } \\
\text { variant } 2\end{array}$ & [GenBank:NM 004355.2] \\
\hline 12 & IQWDI* & IQ motif and WD repeats I & [GenBank:BC025262.1] \\
\hline 13 & APOL3 & Apolipoprotein L3 & [GenBank:AF305227.I] \\
\hline 14 & DUSP3 & Dual specificity phosphatase 3 & [GenBank:BC03570I.I] \\
\hline 15 & SEPT4 & Septin 4, transcript variant I & [GenBank:NM 004574.2] \\
\hline 16 & $\mathrm{CFH}$ & Complement factor $\mathrm{H}$, transcript variant $\mathrm{I}$ & [GenBank:NM 000186.3] \\
\hline 17 & HLA-DPAI & Major histocompatibility complex, class II, DP alpha I & [GenBank:NM 033554.2] \\
\hline 18 & AlFI & Allograft inflammatory factor I & [GenBank:U197|3.I] \\
\hline 19 & $O L R I^{*}$ & Oxidized low density lipoprotein (lectin-like) receptor I & [GenBank:NM 002543.3] \\
\hline 20 & ASPHD2 & Aspartate beta-hydroxylase domain containing 2 & [GenBank:NM 020437.4] \\
\hline 21 & LGALS3BP & Lectin, galactoside-binding, soluble, 3 binding protein & [GenBank:NM 005567.3] \\
\hline 22 & PSMB2 & Proteasome (prosome, macropain) subunit, beta type, 2 & [GenBank:NM 002794.3] \\
\hline 23 & TMSBIO & Thymosin beta 10 & [GenBank:NM 021103.3] \\
\hline 24 & STXII & Syntaxin II & [GenBank:AF044309.I] \\
\hline 25 & ZAK & Sterile alpha motif and leucine zipper containing kinase AZK, transcript variant I & [GenBank:NM 016653.2] \\
\hline 26 & PSMB8 & $\begin{array}{l}\text { Proteasome (prosome, macropain) subunit, beta type, } 8 \text { (large multifunctional } \\
\text { peptidase } 7 \text { ), transcript variant } 2\end{array}$ & [GenBank:NM |48919.3] \\
\hline 27 & MSRB2 & Methionine sulfoxide reductase B2 & [GenBank:NM 012228.3] \\
\hline 28 & HLA-DRB3 & Major histocompatibility complex, class II, DR beta 3 & [GenBank:BC008987.I] \\
\hline 29 & ELMO2 & Engulfment and cell motility 2, transcript variant I & [GenBank:NM 133171.3] \\
\hline 30 & SSB & Sjogren syndrome antigen B (autoantigen La) & [GenBank:NM 003142.3] \\
\hline 31 & UBE2L3 & Ubiquitin-conjugating enzyme UbcH7 & [GenBank:AJ000519.1] \\
\hline 32 & CI 6orf75 (MGC24665) & Chromosome 16 open reading frame 75 & [GenBank:BC022427.I] \\
\hline 33 & AGPAT9 (HMFN0839)* & I-Acylglycerol-3-phosphate O-acyltransferase 9 & [GenBank:NM 0327/7.3] \\
\hline 34 & MTHFD2 & $\begin{array}{l}\text { Methylenetetrahydrofolate dehydrogenase (NADP+ dependent) } 2 \text {, } \\
\text { methenyltetrahydrofolate cyclohydrolase }\end{array}$ & [GenBank:NM 006636.3] \\
\hline 35 & PSMA5 & Proteasome (prosome, macropain) subunit, alpha type, 5 & [GenBank:NM 002790.2] \\
\hline 36 & ZNF28I* & Zinc finger DNA binding protein $99(28 \mathrm{I})$ & [GenBank:AFI25I58.I] \\
\hline 37 & ROBLD3 (MAPBPIP) & Roadblock domain containing 3 & [GenBank:BC024190.2] \\
\hline
\end{tabular}

*Transcripts underexpressed in patients with septicemic melioidosis when compared to sepsis due to other organisms

MAPKK activity. Finally, the list also includes genes that have not previously been associated with the immune response (IQWD1, FAM26F, C16orf75, AGPAT9, and C19orf12).

The results we have obtained were confirmed by quantitative PCR (qPCR) for the top 11 classifiers chosen after ranking the transcripts based on fold change and difference in intensity
(Figure $\mathrm{S}_{5}$ in Additional data file 2). Significant correlation (Pearson correlation test, $\mathrm{r}=0.57$ or higher, $P<0.05$ ) was observed between the expression level determined by microarray and by qPCR in the training $\left(n=24\right.$; Figure $\mathrm{S}_{5}$ a in Additional data file 2) and test set $1\left(n=23\right.$; Figure $\mathrm{S}_{5} \mathrm{~b}$ in Additional data file 2) for all 11 classifiers. 


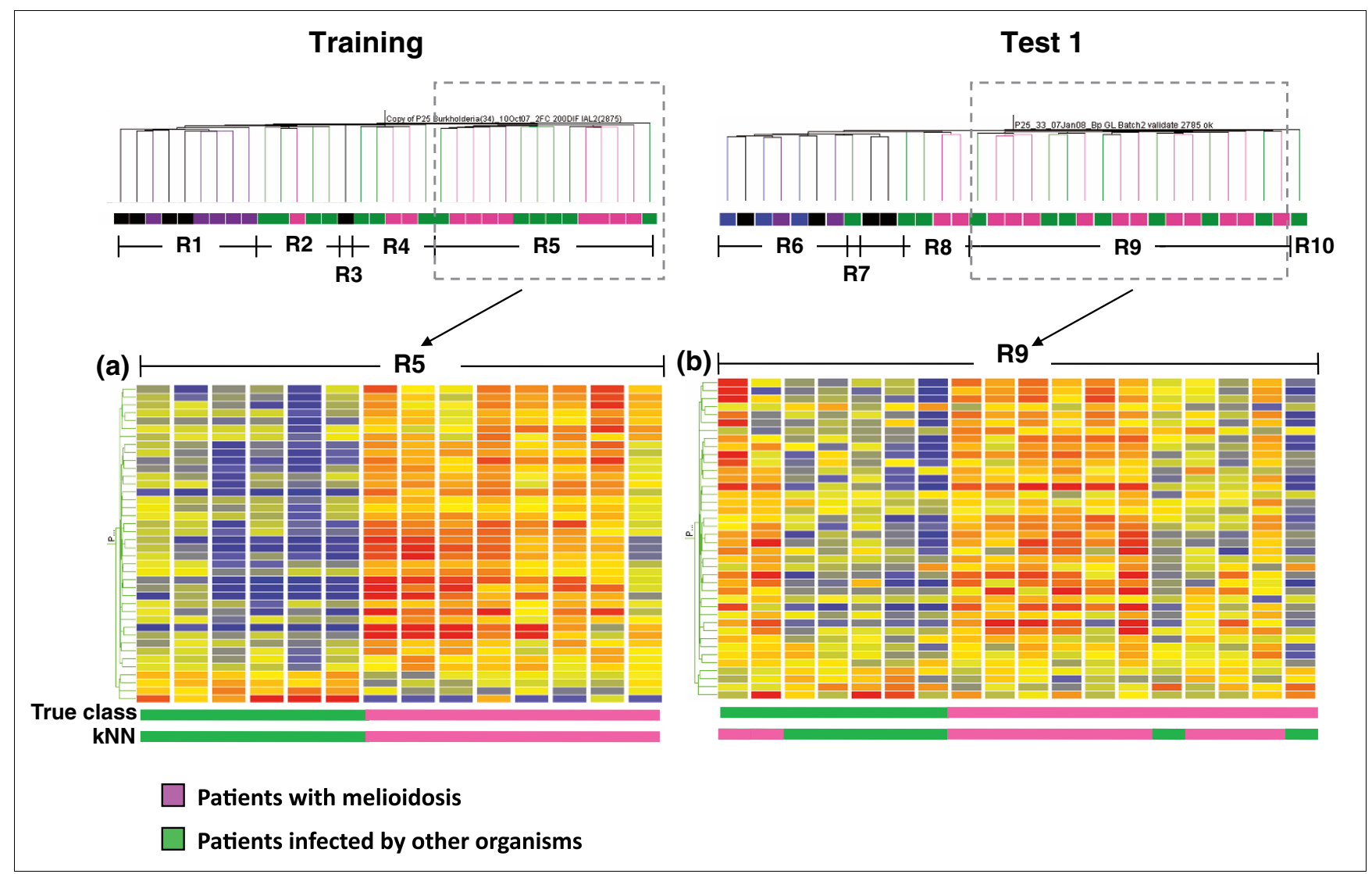

Figure 6

Candidate blood transcriptional markers discriminate sepsis due to B. pseudomallei from sepsis due to other organisms. (a) Patients with sepsis in R5 of the training set (comprising eight patients with melioidosis (pink rectangles) and six patients with sepsis caused by other organisms (green rectangles)) were subjected to class prediction analysis (K-nearest neighbors $(\mathrm{kNN})$ ) using the leave-one-out cross-validation scheme. This algorithm identified $37 \mathrm{classifiers}$ that discriminated samples with 100\% accuracy in the training set. (b) Independent validation of the 37 predictors was performed with the equivalent region $\mathrm{R} 9$ in test set $\mathrm{I}$, including $\mathrm{II}$ patients with melioidosis (pink) and 7 patients with sepsis caused by other organisms (green). The predictors correctly classified 14 of the 18 samples (78\% accuracy).

\section{Secondary validation of the candidate biomarker signature}

The performance of the candidate biomarkers identified in the training set was further evaluated in a second independent set of samples $(n=15)$. This secondary validation was performed using the most recent Illumina expression BeadChip (HumanHT-12 V3). The content of this BeadChip was revised to account for updates made to the National Center for Biotechnology Information Reference Sequence database (NCBI RefSeq) since the release of the version 2 BeadChip. We first generated technical replicates by running the cRNA samples of septic patients in region $\mathrm{R}_{5}(\mathrm{n}=14)$ of our training set on the new BeadChip platform. The set of 37 candidate biomarkers identified from analysis using the Hu6 V2 beadchip (40 probes) were mapped to 47 equivalent probes on the HumanHT-12 V3 BeadChip. Class prediction analysis using these 47 probes classified perfectly samples from patients with septicemic melioidosis and patients with sepsis caused by other organisms (region R5 of the training set; $100 \%$ accuracy; leave-one-out cross-validation; Figure 8a).
This same set of $47 \mathrm{~V}_{3}$ BeadChip probes was then used to classify the 15 samples of the second test set. Consistent with the results obtained in our first test set, the candidate biomarkers efficiently distinguished patients with septicemic melioidosis $(\mathrm{n}=8)$ from those patients with other pathogens $(\mathrm{n}=7)$ with $80 \%$ accuracy (Fisher's exact test, $P$-value $=0.0406$ ) and 3 samples were misclassified (Figure 8b; Table $\mathrm{S}_{4}$ in Additional data file 2). The resulting sensitivity and specificity was 0.71 (exact 95\% confidence interval, 0.29 to 0.96 ) and 0.88 (exact $95 \%$ confidence interval, 0.47 to 0.997 ), respectively.

Thus, class prediction analysis identified and independently validated a candidate blood transcriptional signature for the differential diagnosis of septicemic melioidosis. Furthermore, significant functional convergence was observed among the transcripts forming this signature, which appear to be principally involved in antigen processing and presentation. In the present study, we aimed to compare the signatures of patients with septicemic melioidosis and of patients with sepsis caused by other infections with the goal of identi- 
(a)

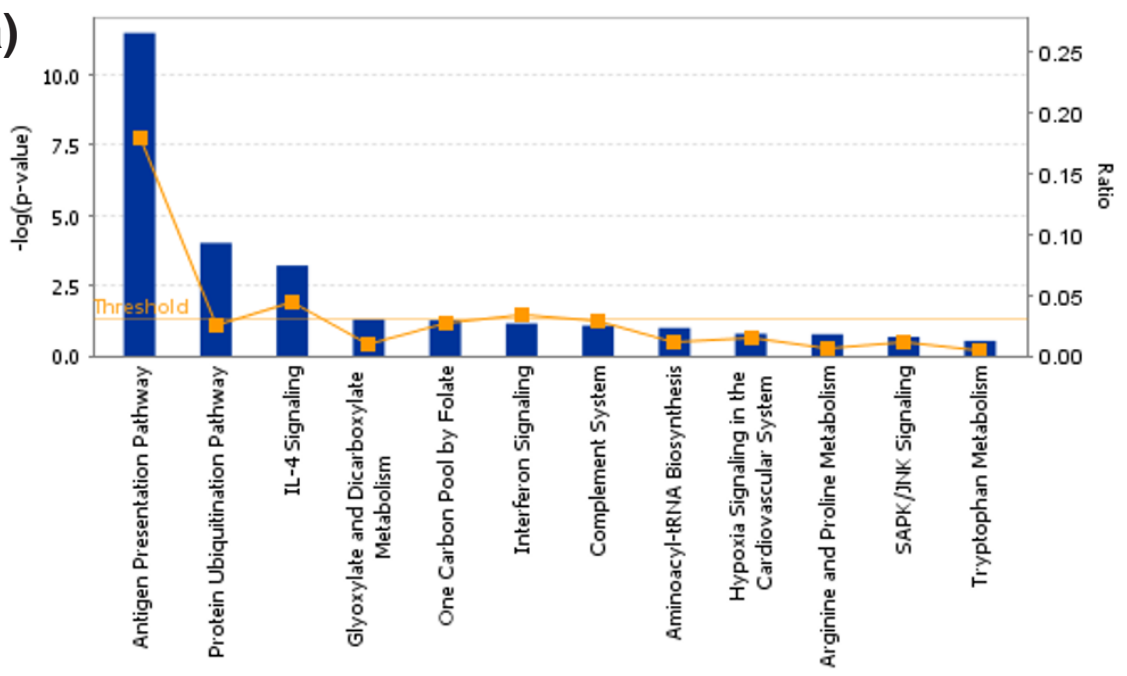

(b)
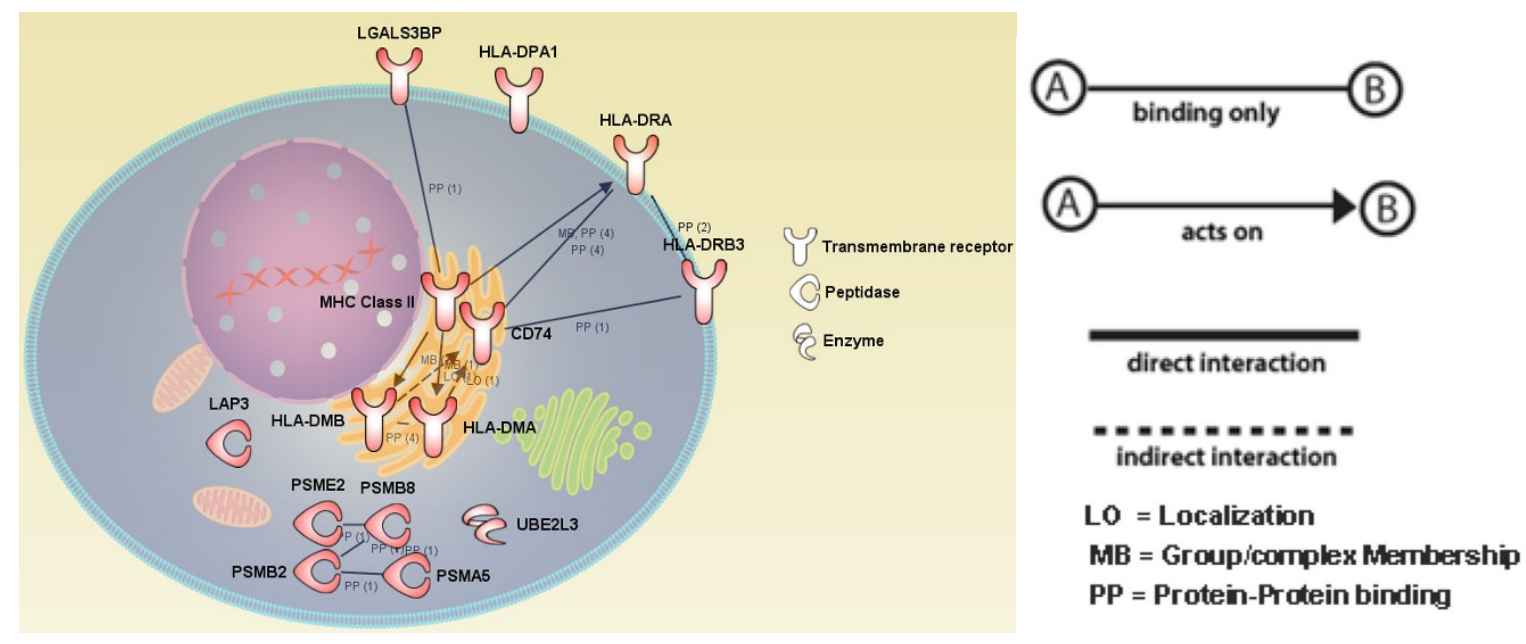

Figure 7

Canonical pathway and gene network analysis of the 37 classifiers. (a) The 37 classifiers were analyzed using ingenuity pathway analysis and the classifiers were grouped to 12 canonical biological process pathways. The antigen presentation pathway ( 7 molecules) and protein ubiquitination pathway ( 5 molecules) were found to be the dominant canonical pathways represented by these set of classifiers. The orange squares indicate the ratio of the number of genes from the dataset that map to the canonical pathway, whilst the solid blue bars correspond to the $P$-value representing the probability that the association between the genes in the classifier set and the identified pathway occurs by chance alone (calculated by Fischer's exact test, and given as a log $P$-value). A representative gene network of the dominant canonical pathways was then generated (b). Transcripts that are overexpressed in patients with melioidosis are indicated by a red color. The function of the gene product is represented by a symbol. Connections between the gene products and the nature of these interactions are shown.

fying candidate biomarkers for the differential diagnosis of melioidosis.

\section{Discussion}

Genome-wide blood transcriptional profiling affords a comprehensive assessment of the immune status of patients. To date, signatures have been reported for a number of systemic diseases, including sepsis [18,22-25,31-35]. A recent report described blood leukocyte mRNA profiles of 35 genes related to inflammation, such as interleukin-1 $\beta$, interferon- $\gamma$, and tumor necrosis factor- $\alpha$, in patients with melioidosis and healthy control subjects [36]. We have extended the findings of this study with the characterization and independent validation of a robust whole blood signature measured on a genome-wide scale ( $>48,000$ probes) in control subjects and in patients with sepsis caused by a wide range of organisms, including $B$. pseudomallei. Whereas all patients with sepsis clearly demonstrated patterns of expression distinct from that of non-infected controls with over 8,00o transcripts found to be differentially expressed, unsupervised analyses also revealed heterogeneity among the sepsis signature. 


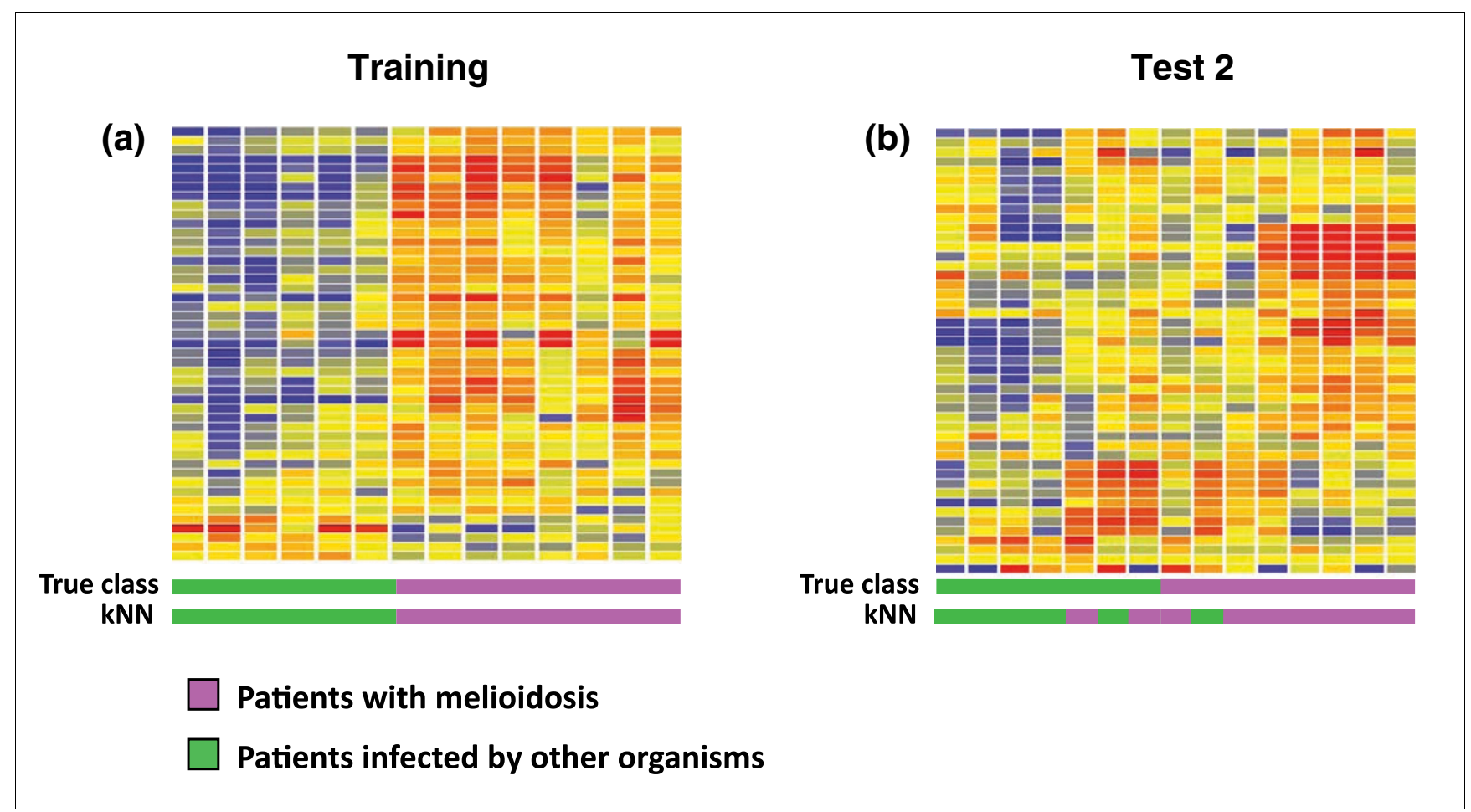

\section{Figure 8}

Candidate blood transcriptional markers retain their discriminatory power in an additional secondary validation set. (a) Patients with sepsis clustered in region R5 of the training set (comprising eight patients with melioidosis (pink rectangles) and six patients with sepsis caused by other organisms (green rectangles) were hybridized to Illumina Human HT-12 V3 BeadChips and used for microarray analysis as before. The 37 blood transcriptional markers identified from the same samples using Illumina Human V2 BeadChips were used for class prediction analysis of the new dataset in a leave-one-out crossvalidation scheme as before. The 37 classifiers discriminated training set samples analyzed using the novel data with $100 \%$ accuracy as before, despite the change of microarray platform. (b) The performance of the 37 predictor genes was then further evaluated in a secondary independent test set also analyzed using Illumina Human HT- 12 V3 BeadChips. This second independent test set $(n=15)$ comprised eight patients with melioidosis (pink rectangles) and seven patients with sepsis caused by other organisms (green rectangles). The predictors correctly classified 12 of the 15 samples ( $80 \%$ accuracy).

Applying a modular analysis framework demonstrated differences at the functional level and a molecular distance metric showed marked differences in the levels of transcriptional perturbations between the different patient clusters. We and others have formerly demonstrated pathogen-specific transcriptional signatures in patients with acute infections, but differences in disease etiology could not explain the heterogeneous signatures observed here. These observations support that the first order of variation in this dataset may originate from differences in disease severity. Longitudinal analyses on samples collected serially should be performed to confirm this hypothesis.

A number of studies have employed gene expression microarrays to measure the responses of host cells to pathogenic microorganisms [19-25,37,38]. Specifically, the analysis of patients' blood leukocyte transcriptional profiles has led to a better understanding of host-pathogen interaction and pathogenesis and yielded distinct diagnostic signatures [3739]. Moreover, others have shown that clinical illness caused by non-infectious causes of systemic inflammatory response syndrome or infection-proven sepsis can be distinguished using the transcriptional signature of peripheral blood mononuclear cells [40]. In addition, illness severity levels and septic shock subclasses of pediatric patients have also been identified through genome-wide expression profiling [41].

Here we report a signature differentiating melioidosis from sepsis caused by other pathogens. Prediction of melioidosis from sepsis caused by other organisms yielded 100\%, 78\%, and $80 \%$ accuracy in the training set and the first and second independent test sets, respectively. The two misclassified patients who were erroneously predicted to belong to the melioidosis group had clinical diagnoses of coagulase-negative staphylococcal (patient I016) and E. coli (patient Io23) septicemia. Patient Io23 had community-acquired septicemia resulting from a leg wound. Patient I016 was hospitalized for 2 weeks prior to the collection of the blood culture from which the coagulase-negative staphylococci were isolated and thus it is plausible that they had true hospitalacquired coagulase-negative staphylococcal septicemia. However, it is equally likely that this isolate was not the true causative agent for the sepsis, in which case it is less surprising that the classification of this sample is incorrect. Coagu- 
lase-negative staphylococci were felt to be the organism responsible for sepsis in at least one patient (Io18), who was a chronic renal failure patient on dialysis. Coagulase-negative staphylococcal bacteremia is more common in such patients due to the need for frequent connection to plastic lines for dialysis [42]. The organism was isolated in two separate sets of blood cultures from this patient, who was then treated with vancomycin and recovered. For other patients with coagulase-negative staphylococcal bacteremia (Io20, Io22), the organism was also isolated from two separate sets of blood cultures, suggesting that, in these cases, coagulase-negative staphylococci may be the true causative pathogen. In the remaining cases, it is possible that the coagulase-negative staphylococci were not the true causative pathogen, but the patients meet the criteria for sepsis and thus still form a useful control group against melioidosis, essentially as a group of patients with 'sepsis of uncertain origin'. This reflects a common and important clinical scenario.

Due to concerns over this possible diagnostic misclassification, however, a second independent test set, with no coagulase-negative staphylococcal bacteremia cases, was also used to validate the findings of the training set. Notably, this study adds a second level of validation that goes beyond the training/independent testing scheme that is starting to appear more commonly in microarray publications. The level of classification accuracy of $80 \%$ observed in our second independent test set confirmed our earlier results. In this last set two patients with sepsis attributed to Corynebacterium spp. (patient Io28) and S. aureus (patient Io29) were misclassified as septicemic melioidosis. These patients stayed in a hospital for more than 10 days before collection of the subsequently positive blood culture. One patient with septicemic melioidosis was erroneously classified as having sepsis caused by another pathogen (patient Mo33).

We report that the 37 classifiers forming the diagnostic signature were significantly enriched in transcripts whose products are involved in class II antigen processing and presentation, including nonclassical MHC molecules $H L A$ $D M A$ and $H L A-D M B$, which catalyze the removal of invariant chain $\mathrm{CD} 74$ from the MHC class II binding groove and facilitate peptide loading to MHC class II molecules within intracellular compartments, as well as classical MHC class II molecules $H L A-D R A,-D R B_{3}$, and -DPA1, which function by the presentation of loading peptides onto the cell surface. Association between $H L A-D R B 1^{*} 1602$ and severe melioidosis in the Thai population has been proposed [43]. Indeed, patients who do not survive sepsis have decreased $H L A-D R A$, -DMA, $-D M B$, and $C D 74$ mRNA expression in whole blood and reduced $H L A-D R$ expression on the cell surface of circulating monocytes $[44,45]$. The numbers of circulating blood dendritic cells has recently been linked to disease severity in septic patients [46]. This study found significantly lower blood myeloid dendritic cell and plasmacytoid dendritic cell counts in septic patients than in controls. Moreover, decreased numbers of circulating of myeloid and plasmacytoid dendritic cells has also been reported to be associated with mortality in patients with septic shock [46]. Since HLADR is a well recognized marker of dendritic cell activation, such findings suggest a possible link between $H L A-D R$ expression level, the number of circulating dendritic cells and disease severity. In the present study, decreased mRNA expression of these transcripts was observed in septic patients compared to uninfected controls. Among septic patients, elevated MHC class II mRNA expression discriminated septicemic melioidosis from other sepsis. A recent study has reported decreased expression of these MHC class II molecules in patients with sepsis [29]. Taken together, measuring the expression of these molecules at the transcriptional or protein levels may be useful for the diagnosis of melioidosis. Transcripts encoding the $20 \mathrm{~S}$ proteasome (PSMB2, PSMB8, PSMA5), 11S activator (PSME2) and $U B E 2 L_{3}$ in the ubiquitin-proteasome pathway, which are responsible for protein degradation and generating pathogen-derived peptides for loading onto MHC class I molecules for presentation to CD8+ $\mathrm{T}$ cells, were also listed as classifiers for the differential diagnosis of melioidosis in the present study. The differential expression of transcripts in this pathway has also been reported in patients with dengue hemorrhagic fever [20]. This pathway is believed to be important in host defense against intracellular pathogens and viruses [47]. Given that B. pseudomallei is an intracellular pathogen, it is biologically plausible that this pathway would have an important role in the host response to melioidosis. Other classifiers found in our study are also involved in immune responses. Increased abundance of $A I M 2$ (interferon-inducible and neutrophil-related gene), $L A P_{3}$ (interferon-inducible gene) and $W A R S$ (interferon-response gene) found in our study has also been observed to be over-expressed in patients with malaria [19]. These transcripts are induced by interferon- $\gamma$, which correlated with our observation of increased abundance of interferon-induced mRNA transcripts (Figure 5, module M3.1) Over-expression of $L G A L S_{3} B P$, which is involved in cell-cell and cell-matrix interaction, was also found in our study. Over-expression of this transcript has been reported in the blood of patients with febrile respiratory illnesses and protein levels have been found to be elevated in the serum of patients with human immunodeficiency virus infection [21,48]. The fact that a significant functional convergence exists among the transcripts forming this diagnostic biomarker signature is important as it suggests that it may be stemming from differences rooted in the pathophysiology of $B$. pseudomallei.

In addition to providing valuable diagnostic information, blood transcriptional assays that measure the host response to infection could potentially serve to monitor disease progression and response to treatment. A test combining such characteristics would contribute to improvements in the management of sepsis. In a context where medical care facilities could be quickly overwhelmed, a test measuring the host response to infection would facilitate early diagnosis and an 
evaluation of disease severity, thus proving to be particularly valuable as a triage tool.

Thus far, several practical considerations have limited the implementation of blood transcriptional testing. Microarray technologies, while constituting an excellent tool in the discovery phase, are currently inadequate for routine testing. Indeed, the data that are generated are not quantitative and are therefore susceptible to batch-to-batch variations. Furthermore, the turnaround time for the processing of samples and generation of data is too long to be of use in a critical care setting. Real-time PCR based assays address such limitations but are only amenable to the quantification of a small number of transcripts. New technologies, however, are becoming available for quantitative 'digital' transcriptional profiling of large sets of genes [49]. An additional advantage of this study is that our findings are based on whole blood transcriptional profiling. This obviates the need for complex additional processing of the blood sample to extract peripheral blood mononuclear cells or other cell fractions or subpopulations, which requires significant laboratory experience and additional equipment. Taken together, the convergence of recent advances made in the collection of blood samples, measurement of transcript abundance and bioinformatics analyses could make clinical translation achievable.

\section{Conclusions}

Microarrays were used to study genome-wide blood transcriptional profiles of patients with sepsis caused by B. pseudomallei. We are reporting the identification of a candidate signature for the differential diagnosis of septicemic melioidosis that classified samples with nearly $80 \%$ accuracy in a first independent test set and $80 \%$ in a second validation set. The molecular distance metric that we describe here for the first time also remains to be evaluated as a potential indicator of disease severity. Finally, the diagnostic signature that we have identified was significantly enriched in genes involved in the MHC class II antigen processing and presentation pathway and the implication of this finding for B. pseudomallei pathogenesis will be the subject of further investigations.

\section{Materials and methods}

\section{Enrolment, sample collection, and informed consent}

A total of 598 subjects consisting of 29 uninfected controls and 569 patients suspected of having contracted communityacquired or nosocomial infection were recruited for this study. Of these subjects, those from whom samples were collected in 2006 and met the enrolment criteria were assigned to the training set whereas those from whom samples were collected in 2007 and 2008 were assigned to test set 1 and test set 2, respectively. Clinical specimens (for example, blood, sputum, urine) were collected for bacterial culture within 24 hours following the diagnosis of sepsis. All blood samples were obtained at the Khon Kaen Regional Hospital, Khon
Kaen, Thailand. Each patient enrolled in the study had three milliliters of whole blood collected into Tempus vacutainer tubes (Applied Biosystems, Foster City, CA, USA) containing an RNA stabilization solution. The tubes were mixed vigorously for 30 seconds to ensure complete sample homogenization. The whole blood lysate was stored at $-80^{\circ} \mathrm{C}$ prior to extraction. Sixty-three of the enrolled patients had the diagnosis of bacteremic sepsis retrospectively confirmed by the isolation of a causative organism on blood culture. Patients who had negative blood cultures were excluded from further study. Community-acquired septicemia was defined when the first positive blood culture was obtained from samples collected within 48 hours of hospitalization, whereas nosocomial septicemia was defined if the infection developed after 48 hours of hospitalization or within 14 days of a previous admission [50].

The diagnosis of sepsis for this study was taken from accepted international guidelines and defined as presentation with two or more of the following criteria for the systemic inflammatory response syndrome: fever (temperature $>38^{\circ} \mathrm{C}$ or $<36^{\circ} \mathrm{C}$ ), tachycardia (heart rate $>90$ beats/minute), leukocytosis or leukocytopenia (white blood cell count $\geq 12 \times 10^{9} / 1$ or $\leq 4 \times 10^{9} / 1$ ) [51]. Severe infection was defined as the presence of systemic hypoperfusion: shock (systolic blood pressure $<90 \mathrm{mmHg}$ or requirement for vasopressors or inotropes for $>1$ hour in the absence of other causes of hypotension), renal dysfunction (oliguria: urine output $<500 \mathrm{ml}$ per 24 hours), liver dysfunction (bilirubin level of $>2.0 \mathrm{mg} / \mathrm{dl}$ ), and thrombocytopenia (platelet count $<100,000$ cells $/ \mathrm{ml}$ ). A total of 92 blood samples from control subjects and septicemic patients that met the case definitions were analyzed, including 63 patients with sepsis ( 32 patients with septicemic melioidosis, 31 patients with sepsis due to other organisms) and 29 noninfected controls (9 patients recovered from melioidosis, 12 patients with T2D, and 8 healthy donors) (Figure 1). Among the sepsis group, 3 whole blood samples were collected before antibiotics were given while 60 whole blood samples were drawn after the start of antibiotic therapy. Two samples were collected after anti-fungal drugs were given. Of 32 patients with melioidosis, 20 (63\%) had pneumonia, a common clinical presentation of the disease. Twelve patients infected by other organisms also had pneumonia (39\%). Clinical information is available in Additional data file 1. The study protocol was approved by the Institutional Review Boards of each participating institution and informed consent was obtained for all subjects.

\section{Microarray assay}

RNA preparation and microarray hybridization

Total RNA was isolated from whole blood lysate using the Tempus Spin Isolation kit (Applied Biosystems) according to the manufacturer's instructions. RNA integrity values were assessed on an Agilent 2100 Bioanalyzer (Agilent, Palo Alto, CA, USA). Samples with RNA integrity values $>6$ were retained for further processing (average RNA integrity values 
$=7.9$, standard deviation $=0.89$ ) . Globin $\mathrm{mRNA}$ was depleted from a portion of each total RNA sample using the GLOBINclear $^{\mathrm{TM}}$-Human kit (Ambion, Austin, TX, USA). Globinreduced RNA was amplified and labeled using the Illumina TotalPrep RNA Amplification Kit (Ambion). Labeled cRNA was hybridized overnight to Sentrix Human-6 V2 or HumanHT-12 V3 expression BeadChip array (IIlumina, San Diego, CA, USA), washed, blocked, stained and scanned on an Illumina BeadStation 500 following the manufacturer's protocols. The dataset described in this manuscript is deposited in the NCBI Gene Expression Omnibus (GEO) with series accession number [GEO:GSE13015].

\section{Microarray data extraction and normalization \\ Microarray data analysis \\ Normalization}

Illumina's BeadStudio version 2 software was used to generate signal intensity values from the scans. After background subtraction, the average normalization recommended by the BeadStudio 2.0 software was used to rescale the difference in overall intensity to the median average intensity for all samples across multiple arrays and chips. After that, the standard normalization procedure for one-color array data in GeneSpring GX7.3 software (Agilent Technologies) was used. In brief, data transformation was corrected for low signal, with intensity values < 10 set to 10 . In addition, per-gene normalization was applied by dividing each probe intensity by the median intensity value for all samples.

\section{Unsupervised analysis}

The aim is to group samples on the basis of their molecular profiles without a priori knowledge of the phenotypic classification. The first step consists in selecting transcripts that are expressed in the dataset, and present some degree of variability: transcripts must have a detection $P$-value less than the $P$ value cutoff of 0.01 in at least two samples (data file filter in GeneSpring GX 7.3); and they must vary by at least two-fold from the median intensity calculated across all samples with a minimum difference $\geq \mathbf{2 0 0}$. The probes passing the filtering criteria were used to group samples in GeneSpring GX 7.3 following two distinct strategies, hierarchical clustering and PCA.

Hierarchical clustering is an iteratively agglomerative clustering method that was performed to find similar transcriptional expression patterns and to produce gene trees or condition trees representing those similarities. The hierarchical clustering performed in our dataset was calculated through the average linkage while the similarity or dissimilarity of gene expression profiles was measured using Pearson correlation, which is the default in the software. By using this algorithm, samples were segregated into distinct groups based on similarity in expression patterns. Gene trees are represented in the horizontal dimension while condition trees are represented in the vertical dimension. The color conventions for all maps are as follows: red indicates over-expressed transcripts, blue underexpressed transcripts, and yellow transcripts that do not deviate from the median.

PCA on conditions was performed to visualize the differences in expression levels of the entire dataset. This approach was performed through JMP genomics software (SAS, Cary, NC, USA) to find and interpret the complex relationships between variables in the dataset from each study group. The first three components, $\mathrm{PC}_{1}, \mathrm{PC}_{2}$ and $\mathrm{PC}_{3}$, were plotted against each other. Each colored dot represents an individual sample.

\section{Supervised analysis}

The aim of the supervised analysis is to identify probes that are differentially expressed between study groups and that might serve as classifiers. We adopted two different strategies for probe selection. First, transcripts that were present in at least two samples in the dataset were selected for statistical group comparison. Second, the parametric Welch $t$-test was used with $P<0.01$ and three levels of stringency for multiple testing correction - Bonferroni, Benjamini and Hochberg, and no multiple testing correction were set for the statistical group comparison (GeneSpring GX 7.3 software).

\section{Class prediction}

Class prediction analyses were carried out to determine whether whole blood from patients with sepsis due to B. pseudomallei infection carry gene expression signatures that can classify them separately from that of whole blood obtained from septic patients caused by other organisms. Significantly different transcripts (parametric Welch $t$-test, $P<0.01$ ) changing by at least 1.5 -fold between the study groups were used as a starting point for the identification of classifiers using the K-nearest neighbors algorithm. This set of classifier genes was validated in an independent group of patients (test sets 1 and 2).

\section{Molecular distance analysis}

This novel approach consists in the computation of a score representing the 'molecular distance' of a given sample relative to a baseline (for example, healthy controls). This approach essentially consists in carrying out outlier analyses on a gene-by-gene basis, where the dispersion of the expression values found in the baseline samples (controls) is used to determine whether the expression value of a single case sample lies inside or outside two standard deviations of the controls' mean. This analysis was performed by merging the transcripts from all modules, which accounted for 2,109 probes. The distance of each sample from the uninfected control baseline was calculated as follows. In step 1 the baseline is established: for each gene the average expression level and standard deviation of the uninfected control group is calculated. In step 2 the 'distance' of an individual gene from the baseline is calculated: the difference in raw expression level from the baseline average of a gene is determined for a given sample, and then the number of standard deviations from baseline levels that the difference in expression represents is 
calculated. In step 3 filters are applied: qualifying genes must differ from the average baseline expression by at least 200 and 2 standard deviations. In step 4 a global distance from baseline is calculated: the number of standard deviations for all qualifying genes is added to yield a single value, the global distance of the sample from the baseline.

\section{Transcriptional module-based analysis}

This mining strategy has been described in detail elsewhere [27]. Briefly, a total of 139 blood leukocyte gene expression profiles were generated using Affymetrix U133A\&B GeneChips (44,760 probe sets). Transcriptional data were obtained for eight experimental groups, including systemic onset juvenile idiopathic arthritis, systematic lupus erythematosus, liver transplant recipients, melanoma patients, and patients with acute infections (E. coli, S. aureus, and influenza A). For each group, transcripts with an absent flag call across all conditions were filtered out. The remaining genes were distributed among 30 sets by hierarchical clustering (k-means algorithm; clusters $\mathrm{C} 1$ through $\mathrm{C}_{3}$ ). The cluster assignment for each gene was recorded in a table and distribution patterns across the eight diseases were compared among all the genes. Modules were selected using an iterative process starting with the largest set of genes that belonged to the same cluster in all study groups (that is, genes that were found in the same cluster in eight of the eight groups). The selection was then expanded to include genes with 7 of 8,6 of 8 and 5 of 8 matches to the core reference pattern. The resulting set of genes from each core reference pattern formed a transcriptional module and was withdrawn from the selection pool. The process was repeated starting with the second largest group of genes, then the third, and so on. This analysis led to the identification of 5,348 transcripts that were distributed among 28 modules. Each module was attributed a unique identifier indicating the round and order of selection (for example, M3.1 was the first module identified in the third round of selection). In the context of the present study, RefSeq IDs were used to match probes between the Affymetrix U133 and Illumina Hu6 platforms. Unambiguous matches were found for 2,109 out of the 5,348 Affymetrix probe sets.

\section{RT-PCR and $q P C R$}

RNA expression of a selection of the predictor genes was determined by qPCR. The same source of RNA used for microarray analysis was reverse-transcribed in a 96-well plate using the High Capacity cDNA Archive kit (Applied Biosystems, San Diego, CA, USA). Real-time PCR was set up with Roche Probes Master reagents and Universal Probe Library hydrolysis probes (Roche Applied Science, Indianapolis, IN, USA). PCRs were performed on the LightCycler 480 (Roche Applied Science). Secondary derivative calculation data were collected and cross point values of the selected predictor genes were normalized to two housekeeping genes (HRPT1 and TBP) [52]. Relative Expression Software Tool (REST ${ }^{\odot}$ ) was used in analyzing both group comparison and individual fold changes [53]. Primer sequences were as follows: $Z A K$
[GenBank:NM 016653.2] forward primer, 5'-tgacagagcagtccaacacc-3', and reverse primer, 5'-acacatcgtcttccgtccat-3'; FAM26F [GenBank:NM 001010919.1] forward primer, 5'ttctgcagctgaaattctgg-3', and reverse primer, 5'-tgcatgctctgtggctttac-3'; LAP3 [GenBank:NM 015907.2] forward primer, 5'-gctggaaagctgagagagactt-3', and reverse primer, 5'-cctgatgcagaccataaaagg-3'; HLA-DMA [GenBank:NM 006120.3] forward primer, 5'-agctgcgctgctacagatg-3', and reverse primer, 5'-tggccacattggagtagga-3'; MYOF [Gen-

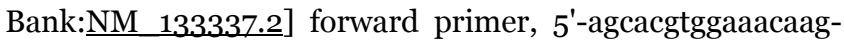
gact-3', and reverse primer, 5'-ccacccacatctgaagttttc-3'; WARS [GenBank:M61715.1] forward primer, 5'-cattttcggcttcactgaca-3', and reverse primer, 5'-gggaatgagttgctgaagga- 3 '; RARRES3 [GenBank:NM 004585.3] forward primer, 5'tgggccctgtatataggagatg-3', and reverse primer, 5 '-ggactgagaagacactggagga-3'; HLA-DMB [GenBank:NM 002118.3] forward primer, 5'-gccttctggggatcact- 3 ', and reverse primer, $5^{\prime}$ tggttttggctacttgcaca-3'; PSME2 [GenBank:NM 002818.2] forward primer, 5'-gggaatgagaaagtcctgtcc- 3 ', and reverse primer, 5'-tcaatcttggggatcaggtg-3'; HLA-DRA [GenBank:NM 019111.3] forward primer, 5'-caagggattgcgcaaaag3', and reverse primer, 5'aagcagaagtttcttcagtgatctt-3'; LGALS3BP [GenBank:NM 005567.3] forward primer, 5'tgtggtctgcaccaatgaa-3', and reverse primer, 5 '-ccgctggctgtcaaagat-3'.

\section{Abbreviations}

MHC: major histocompatibility complex; NIAID: National Institute of Allergy and Infectious Diseases; PC: principal component; PCA: principal component analysis; qPCR: quantitative PCR; T2D: type 2 diabetes.

\section{Authors' contributions}

RP designed the research, performed the research, analyzed the data, and wrote the paper; SB supported the clinical data; MB wrote the paper; DB performed statistical analysis; GJB designed the research; JB designed the research; GL designed the research; and DC designed the research, analyzed the data, and wrote the paper.

\section{Additional data files}

The following additional data are available with the online version of this paper: a table containing specific information regarding individual patients enrolled in this study (Additional data file 1); a document containing Figures $\mathrm{S}_{1}$ to $\mathrm{S}_{5}$ and Tables S1 to S4 (Additional data file 2).

\section{Acknowledgements}

This work was funded by the Baylor Health Care System foundation and the National Institutes of Health (UI9 AIO57234-02 and UOI AI082I 10 to JB) and the NIAID/NIH (AI-6I363). RP was supported by the Commission of Higher Education, Ministry of Education PhD scholarship through Naresuan University, Thailand. We thank the staff of Khon Kaen Regional Hospital for patient recruitment; Quynh-Anh Nguyen, Indira Munagala, 
Chontida Supaprom, Maneerat Pinsiri, and Patcharaporn Tippayawat for their assistance throughout this project; Gordon Hayward for his help uploading the data into GEO; and Charles Quinn for setting up the companion website.

\section{References}

I. Cheng AC, Currie BJ: Melioidosis: epidemiology, pathophysiology, and management. Clin Microbiol Rev 2005, I8:383-4I6.

2. Vuddhakul $V$, Tharavichitkul $P, N a-N g a m ~ N$, Jitsurong $S$, Kunthawa $B$, Noimay P, Binla A, Thamlikitkul V: Epidemiology of Burkholderia pseudomallei in Thailand. Am J Trop Med Hyg 1999, 60:458-46I.

3. Currie BJ: Melioidosis: an important cause of pneumonia in residents of and travellers returned from endemic regions. Eur Respir J 2003, 22:542-550.

4. Stone R: Infectious disease. Racing to defuse a bacterial time bomb. Science 2007, 317:1022-1024.

5. Chaowagul W, White NJ, Dance DA, Wattanagoon Y, Naigowit P, Davis TM, Looareesuwan S, Pitakwatchara N: Melioidosis: a major cause of community-acquired septicemia in northeastern Thailand. J Infect Dis 1989, 159:890-899.

6. White NJ: Melioidosis. Lancet 2003, 36 I:1715-I722.

7. Supaprom C, Wang D, Leelayuwat C, Thaewpia W, Susaengrat W, Koh V, Ooi EE, Lertmemongkolchai G, Liu Y: Development of realtime PCR assays and evaluation of their potential use for rapid detection of Burkholderia pseudomallei in clinical blood specimens. I Clin Microbiol 2007, 45:2894-290I.

8. Chantratita N, Wuthiekanun V, Limmathurotsakul D, Thanwisai A, Chantratita W, Day NP, Peacock SJ: Prospective clinical evaluation of the accuracy of I 6S rRNA real-time PCR assay for the diagnosis of melioidosis. Am J Trop Med Hyg 2007, 77:8I 4-8I7.

9. Meumann EM, Novak RT, Gal D, Kaestli ME, Mayo M, Hanson JP, Spencer E, Glass MB, Gee JE, Wilkins PP, Currie BJ: Clinical evaluation of a type III secretion system real-time PCR assay for diagnosing melioidosis. J Clin Microbiol 2006, 44:3028-3030.

10. Wiersinga WJ, Poll T van der, White NJ, Day NP, Peacock SJ: Melioidosis: insights into the pathogenicity of Burkholderia pseudomallei. Nat Rev Microbiol 2006, 4:272-282.

II. Simpson AJ, Opal SM, Angus BJ, Prins JM, Palardy JE, Parejo NA, Chaowagul W, White NJ: Differential antibiotic-induced endotoxin release in severe melioidosis. I Infect Dis 2000, $181: 1014-1019$

12. Simpson AJ, Suputtamongkol $Y$, Smith MD, Angus BJ, Rajanuwong A, Wuthiekanun V, Howe PA, Walsh AL, Chaowagul W, White NJ: Comparison of imipenem and ceftazidime as therapy for severe melioidosis. Clin Infect Dis 1999, 29:38I-387.

13. Inglis TJ, Rolim DB, Rodriguez JL: Clinical guideline for diagnosis and management of melioidosis. Rev Inst Med Trop Sao Paulo 2006, 48:1-4.

14. Garey KW, Rege M, Pai MP, Mingo DE, Suda KJ, Turpin RS, Bearden DT: Time to initiation of fluconazole therapy impacts mortality in patients with candidemia: a multi-institutional study. Clin Infect Dis 2006, 43:25-3I.

15. Sivalingam SP, Sim SH, Jasper LC, Wang D, Liu Y, Ooi EE: Pre- and post-exposure prophylaxis of experimental Burkholderia pseudomallei infection with doxycycline, amoxicillin/clavulanic acid and co-trimoxazole. J Antimicrob Chemother 2008, 61:674-678.

16. Centers for Disease Control and Prevention [http:// www.emergency.cdc.gov/]

17. Glas AM, Floore A, Delahaye LJ, Witteveen AT, Pover RC, Bakx N, Lahti-Domenici JS, Bruinsma TJ, Warmoes MO, Bernards R, Wessels LF, Van't Veer LJ: Converting a breast cancer microarray signature into a high-throughput diagnostic test. BMC Genomics 2006, 7:278.

18. Allantaz F, Chaussabel D, Banchereau J, Pascual V: Microarraybased identification of novel biomarkers in IL-I-mediated diseases. Curr Opin Immunol 2007, 19:623-632.

19. Griffiths MJ, Shafi MJ, Popper SJ, Hemingway CA, Kortok MM, Wathen A, Rockett KA, Mott R, Levin M, Newton CR, Marsh K, Relman DA, Kwiatkowski DP: Genomewide analysis of the host response to malaria in Kenyan children. J Infect Dis 2005, 191:1599-1611.

20. Simmons CP, Popper S, Dolocek C, Chau TN, Griffiths M, Dung NT, Long TH, Hoang DM, Chau NV, Thao le TT, Hien TT, Relman DA, Farrar J: Patterns of host genome-wide gene transcript abun- dance in the peripheral blood of patients with acute dengue hemorrhagic fever. J Infect Dis 2007, 195: 1097-I I07.

21. Thach DC, Agan BK, Olsen C, Diao J, Lin B, Gomez J, Jesse M, Jenkins M, Rowley R, Hanson E, Tibbetts C, Stenger DA, Walter E: Surveillance of transcriptomes in basic military trainees with normal, febrile respiratory illness, and convalescent phenotypes. Genes Immun 2005, 6:588-595.

22. Ramilo O, Allman W, Chung W, Mejias A, Ardura M, Glaser C, Wittkowski KM, Piqueras B, Banchereau J, Palucka AK, Chaussabel D: Gene expression patterns in blood leukocytes discriminate patients with acute infections. Blood 2007, 109:2066-2077.

23. Prucha M, Ruryk A, Boriss H, Moller E, Zazula R, Herold I, Claus RA Reinhart KA, Deigner $P$, Russwurm S: Expression profiling: toward an application in sepsis diagnostics. Shock 2004, 22:29-33.

24. Shanley TP, Cvijanovich N, Lin R, Allen GL, Thomas NJ, Doctor A, Kalyanaraman M, Tofil NM, Penfil S, Monaco M, Odoms K, Barnes M, Sakthivel B, Aronow BJ, Wong HR: Genome-level longitudinal expression of signaling pathways and gene networks in pediatric septic shock. Mol Med 2007, 13:495-508.

25. Pachot A, Lepape A, Vey S, Bienvenu J, Mougin B, Monneret G: Systemic transcriptional analysis in survivor and non-survivor septic shock patients: a preliminary study. Immunol Lett 2006, 106:63-7I.

26. Huang da W, Sherman BT, Lempicki RA: Systematic and integrative analysis of large gene lists using DAVID bioinformatics resources. Nat Protoc 2009, 4:44-57.

27. Chaussabel D, Quinn C, Shen J, Patel P, Glaser C, Baldwin N, Stichweh D, Blankenship D, Li L, Munagala I, Bennett L, Allantaz F, Mejias A, Ardura M, Kaizer E, Monnet L, Allman W, Randall H, Johnson D, Lanier A, Punaro M, Wittkowski KM, White P, Fay J, Klintmalm G, Ramilo O, Palucka AK, Banchereau J, Pacual V: A modular analysis framework for blood genomics studies: application to systemic lupus erythematosus. Immunity 2008, 29:150-164.

28. Ardura MI, Banchereau R, Mejias A, Di Pucchio T, Glaser C, Allantaz F, Pascual V, Banchereau J, Chaussabel D, Ramilo O: Enhanced monocyte response and decreased central memory $T$ cells in children with invasive Staphylococcus aureus infections. PLoS One 2009, 4:e5446.

29. Wong HR, Cvijanovich N, Allen GL, Lin R, Anas N, Meyer K, Freishtat RJ, Monaco M, Odoms K, Sakthivel B, Shanley TP: Genomic expression profiling across the pediatric systemic inflammatory response syndrome, sepsis, and septic shock spectrum. Crit Care Med 2009, 37:1558-1566.

30. Baylor Institute for Immunology Research [https:// www.biir.net/modules/modules.html]

31. Bennett L, Palucka AK, Arce E, Cantrell V, Borvak J, Banchereau J, Pascual V: Interferon and granulopoiesis signatures in systemic lupus erythematosus blood. J Exp Med 2003, 197:7II-723.

32. Kaizer EC, Glaser CL, Chaussabel D, Banchereau J, Pascual V, White PC: Gene expression in peripheral blood mononuclear cells from children with diabetes. J Clin Endocrinol Metab 2007, 92:3705-37II.

33. Tang BM, McLean AS, Dawes IW, Huang SJ, Cowley MJ, Lin RC: Gene-expression profiling of gram-positive and gram-negative sepsis in critically ill patients. Crit Care Med 2008, 36: I I25-11 28.

34. Wong HR, Shanley TP, Sakthivel B, Cvijanovich N, Lin R, Allen GL, Thomas NJ, Doctor A, Kalyanaraman M, Tofil NM, Penfil S, Monaco M, Tagavilla MA, Odoms K, Dunsmore K, Barnes M, Aronow BJ: Genome-level expression profiles in pediatric septic shock indicate a role for altered zinc homeostasis in poor outcome. Physiol Genomics 2007, 30: I46-I55.

35. Cvijanovich N, Shanley TP, Lin R, Allen GL, Thomas NJ, Checchia P, Anas N, Freishtat RJ, Monaco M, Odoms K, Sakthivel B, Wong HR: Validating the genomic signature of pediatric septic shock. Physiol Genomics 2008, 34: I27-I 34.

36. Wiersinga WJ, Dessing MC, Kager PA, Cheng AC, Limmathurotsakul D, Day NP, Dondorp AM, Poll T van der, Peacock SJ: High-throughput mRNA profiling characterizes the expression of inflammatory molecules in sepsis caused by Burkholderia pseudomallei. Infect Immun 2007, 75:3074-3079.

37. Jenner RG, Young RA: Insights into host responses against pathogens from transcriptional profiling. Nat Rev Microbiol 2005, 3:28I-294.

38. Feezor RJ, Cheng A, Paddock HN, Baker HV, Moldawer LL: Functional genomics and gene expression profiling in sepsis: 
beyond class prediction. Clin Infect Dis 2005, 4I(Suppl 7):S427-435

39. Calvano SE, Xiao W, Richards DR, Felciano RM, Baker HV, Cho RJ, Chen RO, Brownstein BH, Cobb JP, Tschoeke SK, Miller-Graziano C, Moldawer LL, Mindrinos MN, Davis RW, Tompkins RG, Lowry SF: A network-based analysis of systemic inflammation in humans. Nature 2005, 437: 1032-1037.

40. Tang BM, McLean AS, Dawes IW, Huang SJ, Lin RC: Gene-expression profiling of peripheral blood mononuclear cells in sepsis. Crit Care Med 2009, 37:882-888.

41. Wong HR, Cvijanovich N, Lin R, Allen GL, Thomas NJ, Willson DF, Freishtat RJ, Anas N, Meyer K, Checchia PA, Monaco M, Odom K, Shanley TP: Identification of pediatric septic shock subclasses based on genome-wide expression profiling. BMC Med 2009, 7:34.

42. Klevens RM, Edwards JR, Andrus ML, Peterson KD, Dudeck MA, Horan TC: Dialysis Surveillance Report: National Healthcare Safety Network (NHSN)-data summary for 2006. Semin Dial 2008, 2 I:24-28.

43. Dharakul T, Vejbaesya S, Chaowagul W, Luangtrakool P, Stephens HA, Songsivilai S: HLA-DR and -DQ associations with melioidosis. Hum Immunol 1998, 59:580-586.

44. Pachot A, Monneret G, Brion A, Venet F, Bohe J, Bienvenu J, Mougin $B$, Lepape A: Messenger RNA expression of major histocompatibility complex class II genes in whole blood from septic shock patients. Crit Care Med 2005, 33:31-38. discussion 236-237.

45. Lekkou A, Karakantza M, Mouzaki A, Kalfarentzos F, Gogos CA: Cytokine production and monocyte HLA-DR expression as predictors of outcome for patients with communityacquired severe infections. Clin Diagn Lab Immunol 2004, I I:161-167.

46. Guisset O, Dilhuydy MS, Thiebaut R, Lefevre J, Camou F, Sarrat A, Gabinski C, Moreau JF, Blanco P: Decrease in circulating dendritic cells predicts fatal outcome in septic shock. Intensive Care Med 2007, 33:| 48-I52.

47. Strehl B, Joeris T, Rieger M, Visekruna A, Textoris-Taube K, Kaufmann SH, Kloetzel PM, Kuckelkorn U, Steinhoff U: Immunoproteasomes are essential for clearance of Listeria monocytogenes in nonlymphoid tissues but not for induction of bacteria-specific CD8+ T cells. J Immunol 2006, I 77:6238-6244.

48. Darcissac EC, Vidal V, De La Tribonniere X, Mouton Y, Bahr GM: Variations in serum IL-7 and $90 \mathrm{~K} / \mathrm{Mac}-2$ binding protein (Mac-2 BP) levels analysed in cohorts of HIV-I patients and correlated with clinical changes following antiretroviral therapy. Clin Exp Immunol 200I, 1 26:287-294.

49. Geiss GK, Bumgarner RE, Birditt B, Dahl T, Dowidar N, Dunaway DL, Fell HP, Ferree S, George RD, Grogan T, James J], Maysuria M, Mitton JD, Oliveri P, Osborn JL, Peng T, Ratcliffe AL, Webster PJ, Davidson $\mathrm{EH}$, Hood L: Direct multiplexed measurement of gene expression with color-coded probe pairs. Nat Biotechnol 2008 , 26:317-325

50. Friedman ND, Kaye KS, Stout JE, McGarry SA, Trivette SL, Briggs JP, Lamm W, Clark C, MacFarquhar J, Walton AL, Reller LB, Sexton DJ: Health care-associated bloodstream infections in adults: a reason to change the accepted definition of communityacquired infections. Ann Intern Med 2002, I37:791-797.

5I. Dellinger RP, Levy MM, Carlet JM, Bion J, Parker MM, Jaeschke R, Reinhart K, Angus DC, Brun-Buisson C, Beale R, Calandra T, Dhainaut JF, Gerlach H, Harvey M, Marini JJ, Marshall J, Ranieri M, Ramsay G, Sevransky J, Thompson BT, Townsend S, Vender JS, Zimmerman JL, Vincent JL: Surviving Sepsis Campaign: international guidelines for management of severe sepsis and septic shock: 2008. Crit Care Med 2008, 36:296-327.

52. Luu-The V, Paquet N, Calvo E, Cumps J: Improved real-time RTPCR method for high-throughput measurements using second derivative calculation and double correction. Biotechniques 2005, 38:287-293.

53. Pfaffl MW, Horgan GW, Dempfle L: Relative expression software tool (REST) for group-wise comparison and statistical analysis of relative expression results in real-time PCR. Nucleic Acids Res 2002, 30:e36. 